WHOI-89-46

IMET TR-89-03

\title{
Improved Meteorological Measurements from Buoys and Ships (IMET): Preliminary Comparison of Solar Radiation Air Temperature Shields
}

\author{
by
}

Gennaro H. Crescenti, Richard E. Payne and Robert A. Weller

Woods Hole Oceanographic Institution
Woods Hole, Massachusetts 02543

October 1989

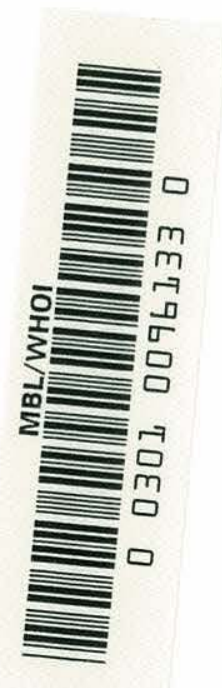
Funding was provided by the National Science Foundation under Grant No. OCE-87-09614

Reproduction in whole or in part is permitted for any purpose of the United States Government. This report should be cited as: Woods Hole Oceanog. Inst. Tech. Rept., WHOI-89-46, IMET TR-89-03.

Approved for publication; distribution unlimited.

Approved for Distribution:

\section{Technical Report}

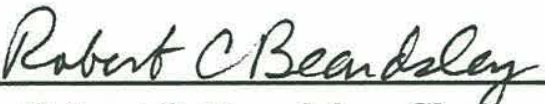

Robert C. Beardsley, Chairman

Department of Physical Oceanography 


\section{Abstract}

Several different types of solar radiation air temperature shields are evaluated for use at sea on ships and buoys. They include three types of static or Thaller shields, two vane oriented shields, and two fan ventilated shields. A preliminary data analysis is presented and discussed. 


\section{Table of Contents}

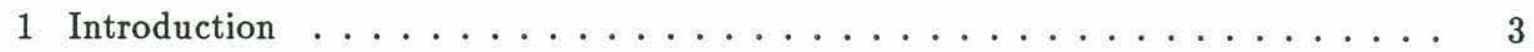

2 Description of Radiation Shields $\ldots \ldots \ldots \ldots \ldots \ldots$

$2.1 \quad$ Static Shields . . . . . . . . . . . . . . . . . 3

$2.2 \quad$ Vane-Oriented Shields . . . . . . . . . . . . . . . 4

$2.3 \quad$ Forced Ventilation $\ldots \ldots \ldots \ldots \ldots \ldots \ldots \ldots \ldots$

3 Data......................... 5

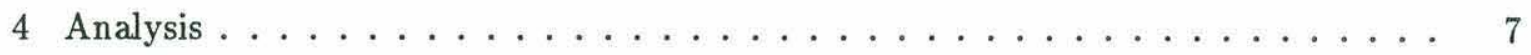

4.1 Plastic Multi-Plate vs. Plastic Multi-Plate . . . . . . . . 7

4.2 Plastic Multi-Plate vs. Aluminum Multi-Plate $\ldots \ldots \ldots \ldots$

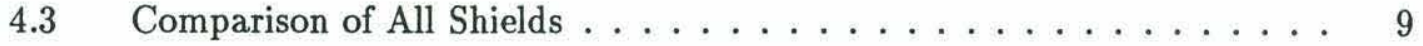

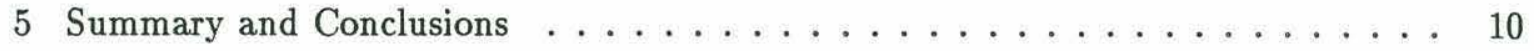

Acknowledgements . . . . . . . . . . . . . . . . . . . 12

References ........................ 13

Appendix A - Manufacturer Addresses . . . . . . . . . . . . . . . 15

Tables . . . . . . . . . . . . . . . . . . . . . . 16

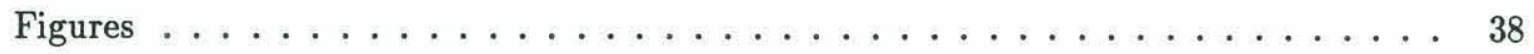




\section{Introduction}

Several components of the U.S. effort in the World Ocean Circulation Experiment (WOCE) will require observations of air-sea heat and momentum fluxes from both ships and buoys. A group at the Woods Hole Oceanographic Institution (WHOI) is developing instrumentation to provide Improved Meteorological measurements (IMET) to meet the requirements of these WOCE components. Here we report on one part of our sensor investigations.

The problems of measuring true ambient air temperature (and true ambient relative humidity) have long been recognized (e.g., Franssila, 1961; Pech, 1964; MacHattie, 1965; Fuchs and Tanner, 1965; Hadlock et al., 1972; Sparks, 1972; McKay and McTaggart-Cowan, 1977). The problem is to protect the sensor completely from solar radiation while allowing sufficient ventilation for the sensor to remain at ambient air temperature. Two approaches have been taken toward providing the ventilation: natural (wind) and forced (fan). Each has its limitations. On a calm sunny day a naturally ventilated shield will not be ventilated sufficiently and its internal temperature will rise no matter how good the shielding is. Ventilation by a fan requires too much power to be used on buoys.

\section{Description of Radiation Shields}

The types of shields investigated thus far are: Natural ventilation

- Static - Three types of Thaller shields differing in construction details.

- Vane-oriented - Two types differing in materials and dimensions.

Forced ventilation

- Two types of fan-ventilated shields.

\subsection{Static Shields}

All three static shields have evolved from a design by Gill (1979) for the National Data Buoy Center. The original specifications called for a shield which would be effective, rugged, and compact. Previous studies (Payne, 1987; 1988) have showed that this type of shield is 
effective at wind speeds above about $2 \mathrm{~m} \mathrm{~s}^{-1}$. Intuitively one would expect the positive temperature bias to increase with increasing irradiance and to decrease with increasing wind speed. The previous investigation (Payne, 1987) showed that the wind speed effect was probably weak but, because of the high correlation between solar radiation and wind speed in the sea breeze-dominated local meteorology, the two effects could not be separated quantitatively.

The R. M. Young Gill multi-plate radiation shield (model 41002) comprises a stack of twelve opaque white injection molded plastic plates (Figure 1). R. M. Young states that with maximum solar radiation, the root mean square errors are $0.4^{\circ} \mathrm{C}$ at $3 \mathrm{~m} \mathrm{~s}^{-1}, 0.7^{\circ} \mathrm{C}$ at $2 \mathrm{~m} \mathrm{~s}^{-1}$, and $1.5^{\circ} \mathrm{C}$ at $1 \mathrm{~m} \mathrm{~s}^{-1}$. Two types were tested. Early models mounted directly above the supporting surface. Recently R. M. Young has changed to an offset mount.

A shield of similar design, but with nine plates of $0.8 \mathrm{~mm}$ thick aluminum, pressure formed, and painted with white Imron, has been developed at WHOI by J. Dean. This shield has been used on some previous buoy deployments (Dean and Beardsley, 1988).

A plastic Gill multi-plate shield was modified during testing by removing the top plate of the shield and replacing it with a solar powered fan.

\subsection{Vane-Oriented Shields}

Vane-oriented shields are being considered as an alternative to static radiation shields. In general, a vane-oriented shield consists of two or more concentric tubes oriented by a tail fin to face the wind at all times when the wind is strong enough to overcome the bearing friction. The temperature sensor is located at the center of the innermost tube. Even in light wind conditions, the motion of the buoy may be sufficient to allow some ventilation.

Two vane-oriented shields are currently under test. The Met One vane radiation shield (model 071A) is constructed of two concentric aluminum tubes (Figure 2). The outer tube has a $76 \mathrm{~mm}$ diameter and is painted with a high gloss white enamel. The vane rotates on two sealed ball bearings and is oriented into the wind by a large tail fin. Past tests have shown that the ball bearings used by the manufacturer corrode in a salt environment. They are probably insufficiently protected to provide reliable operation at sea. 
A prototype shield developed at WHOI by J. Dean has undergone several engineering modifications since Payne's (1987) test (Figure 3). Similar in design to the Met One, it is constructed of three concentric plastic tubes but has a smaller tail fin. The outer tube is $89 \mathrm{~mm}$ diameter, is approximately $50 \mathrm{~cm}$ long, and is painted with white enamel.

\subsection{Forced Ventilation}

Radiation shields that use fans for ventilation are being considered for use on ships where power limitations are not as restrictive as on a buoy. A fan constantly draws in ambient air through several concentric, usually vertically oriented, tubes. In general, higher ventilation rates keep the air temperature inside the shield closer to ambient.

The Met One radiation shield (model 076) draws in ambient air at approximately $2.5 \mathrm{~m} \mathrm{~s}^{-1}$ through the bottom of three concentric aluminum cylinders and is exhausted at the top of the shield (Figure 4). The outside tube is approximately $89 \mathrm{~mm}$ in diameter and is painted with a white enamel.

An R. M. Young Gill Aspirated radiation shield (model 43404) was included for comparison purposes only (Figure 5). This model is obsolete but has been included in past studies (e.g., Payne, 1987). The temperature sensor is mounted inside a vertically-oriented double-walled evacuated silvered glass cylinder. A fan draws in ambient air through the bottom of the tube past the sensor at a rate of approximately $3 \mathrm{~m} \mathrm{~s}^{-1}$. A prototype of a new ventilated shield by R. M. Young is now being tested.

Table 1 contains a summary of the manufacturer specifications for these shields.

\section{Data}

The radiation shields were located on the meteorology tower and on an adjacent aluminum rack on top of the Smith Building of WHOI in Woods Hole, Massachusetts $\left(41^{\circ} 31.5^{\prime} \mathrm{N}, 70^{\circ} 40.0^{\prime} \mathrm{W}\right)$. The building is located on Woods Hole Harbor.

Air temperature is measured with standard YSI thermistors (P/N 44032) (Payne, 1974). The thermistors have a nominal resistance of $30.00 \mathrm{kohms}$ at $20^{\circ} \mathrm{C}$ and are potted in $1 / 4$ inch stainless steel bolts at the factory (probe style 095). The thermistor and bolt assembly are 
then mounted in a protective Delrin cylinder at WHOI. There is approximately $1.6 \mathrm{~mm}$ of plastic between the bolt and ambient air.

The thermistors are calibrated to an accuracy of $0.01^{\circ} \mathrm{C}$ absolute (Payne et al., 1976) at a WHOI calibration facility before each deployment or test. Thermistor drifts are typically less than three millidegrees per year. A precision voltage is applied across the thermistor and a fixed resistor in series. The temperature can be calculated from the voltage drop across the thermistor.

The radiation shield performance was studied in three separate tests. The first test was a comparison of air temperature measurements between two identical R. M. Young Gill multi-plate shields. The shields used the old style mounts which positioned the shields directly above the supporting surface and were spaced approximately $0.5 \mathrm{~m}$ apart. Data were recorded by a NEC APC-IV 286 computer with an Analog Devices RTI-800, 12-bit, analog-to-digital (A/D) board. Data were sampled once every 30 seconds and were averaged and recorded at 7.5 minute intervals. In addition, insolation values were recorded using an Eppley Precision Spectral Pyranometer (model PSP) and wind speed with an R. M. Young Wind Monitor (model 5103).

The second test was a comparison of air temperature measurements between a plastic multi-plate shield and an aluminum multi-plate shield made at WHOI. For this test, data were obtained by two Vector Averaging Wind Recorders (VAWR) (Payne, 1974) placed on the meteorology tower approximately $2 \mathrm{~m}$ apart. The averaging period for these instruments was also 7.5 minutes. Midway through the comparison, the top plate of the plastic shield was removed and replaced with a solar powered fan. Insolation data were recorded using an Eppley Black and White Pyranometer (model 8-48) and wind speed with an aluminum cup anemometer.

The third test was a comparison of all the shields described in section 2. All of these shields were located within about $1 \mathrm{~m}$ of each other. These data were recorded on a NEC APC-IV 286 computer with a Metra-Byte DAS-16, 12-bit, A/D board. Data were sampled once every second and running averages were recorded every 7.5 minutes. Insolation was measured by an Eppley PSP and wind speeds by an R. M. Young Propvane. 


\section{Analysis}

\subsection{Plastic Multi-Plate vs. Plastic Multi-Plate}

Weekly means and standard deviations were computed for each air temperature (WHOI serial numbers YSI-10 and YSI-20) and for the difference (Table 2). The overall mean difference between the two air temperatures is $-0.012^{\circ} \mathrm{C}$ with a standard deviation of $0.074^{\circ} \mathrm{C}$. Figure $6 \mathrm{a}$ shows the probability density of the difference. Weekly mean differences seldom exceeded $0.03^{\circ} \mathrm{C}$.

The means and standard deviations for all nighttime data (insolation values less than $5 \mathrm{Wm}^{-2}$ ) are shown in Table 3. The overall mean difference is $-0.007^{\circ} \mathrm{C}$ with a standard deviation of $0.045^{\circ} \mathrm{C}$. Weekly means of the difference never exceed $0.02^{\circ} \mathrm{C}$. The probability density of all nighttime data (Figure 6b) shows very little spread in the temperature differences. Similarly, the means and standard deviations were computed for all daytime data (insolation values greater than $5 \mathrm{Wm}^{-2}$, Table 4). The probability density (Figure 6c) shows a slightly larger spread in air temperature difference than these nighttime data with an overall mean air temperature difference is $-0.017^{\circ} \mathrm{C}$ with a standard deviation of $0.096^{\circ} \mathrm{C}$. The daytime weekly mean differences seldom exceed $0.05^{\circ} \mathrm{C}$.

Table 5 summarizes the mean differences of air temperature for various ranges of wind speed. Table 6 summarizes the frequency distribution of wind speed. An overall mean difference of $0.03^{\circ} \mathrm{C}$ is found when winds were less than 2 to $3 \mathrm{~m} \mathrm{~s}^{-1}$ for all data. The overall mean difference increases to about $0.6^{\circ} \mathrm{C}$ for all daytime data (Tables 7 and 8 ) for the same range of wind speeds. For winds greater than $3 \mathrm{~m} \mathrm{~s}^{-1}$, the overall mean differences become very small, typically on the order of 0.01 to $0.02^{\circ} \mathrm{C}$.

The mean difference in air temperature is small even though differences in individual data records sometimes exceed $0.2^{\circ} \mathrm{C}$. The accuracy of the measured air temperature is approximately $0.1^{\circ} \mathrm{C}$. This takes into account errors in calibration (and drift) of the thermistors, temperature dependent errors in the A/D board, variations in the precision voltage across the thermistor circuit, and random noise signals (e.g., radio interference). The larger differences, when they do exist, occur when the wind is light and insolation is large. 
We assume that differential heating of the meteorology tower and other surrounding fixtures creates these differences.

We conclude that there is no significant difference in measured air temperatures between the two plastic Gill multi-plate shields.

\subsection{Plastic Multi-Plate vs. Aluminum Multi-Plate}

Weekly means and standard deviations for all data for these two shields appear in Table 9. The overall mean air temperature difference (aluminum-plastic) is $-0.021^{\circ} \mathrm{C}$ with a standard deviation of $0.127^{\circ} \mathrm{C}$. Weekly differences sometimes reached $0.10^{\circ} \mathrm{C}$ but were mostly less than $0.03^{\circ} \mathrm{C}$. The probability density of the differences (Figure $7 \mathrm{a}$ ) is much like the distribution found with the two plastic Gill shields.

The overall mean difference of all nighttime data is $-0.018^{\circ} \mathrm{C}$ with a standard deviation of $0.090^{\circ} \mathrm{C}$ (Table 10). Most of the weekly mean differences are typically less than $0.05^{\circ} \mathrm{C}$. The probability density for these data (Fig. 7b) shows a small spread in temperature differences. The overall mean temperature difference for all daytime data is $-0.023^{\circ} \mathrm{C}$ with a standard deviation of $0.145^{\circ} \mathrm{C}$ (Table 11). Most of the weekly mean differences are less than $0.05^{\circ} \mathrm{C}$ but are sometimes as large as $0.15^{\circ} \mathrm{C}$.

Mean temperature differences were categorized according to wind speed ranges for all data (Tables 12 and 13) and for all daytime data (Tables 14 and 15). In general, the overall mean differences are less than $0.1^{\circ} \mathrm{C}$ in both cases, with the largest weekly mean differences occurring at the lowest wind speeds (less than $2 \mathrm{~m} \mathrm{~s}^{-1}$ ).

Accuracy of temperature measurements with the VAWR are approximately $0.01^{\circ} \mathrm{C}$ (Payne, 1974). Like the first comparison, the mean differences are of the same order as the overall system accuracy of the VAWR. Hence, we see no significant difference between the two shields. The standard deviations, however, are slightly larger, indicating more scatter in these data than with the two plastic Gill shields.

The plastic radiation shield was modified part way through VAWR testing. The top plate of the shield was removed and replaced with a solar powered fan to provide additional ventilation. 
The overall mean difference is $-0.062^{\circ} \mathrm{C}$ with a standard deviation of $0.154^{\circ} \mathrm{C}$ (Table 16). For all the weekly mean differences, the newly modified shield had warmer air temperatures ranging from 0.02 to $0.10^{\circ} \mathrm{C}$. Figure $8 \mathrm{a}$ shows the probability density for these data. Most of the temperature differences occur on the left side of the zero line indicating that the aluminum shield is keeping the air temperature cooler than the plastic shield. The overall mean difference for nighttime data is $-0.005^{\circ} \mathrm{C}$ with a standard deviation of $0.059^{\circ} \mathrm{C}$ (Table 17). The probability density (Figure $8 \mathrm{~b}$ ) is much like the other evening distributions showing very little scatter. The overall mean difference for the all daytime data is $-0.114^{\circ} \mathrm{C}$ with a standard deviation of $0.168^{\circ} \mathrm{C}$ (Table 18). The probability density (Figure 8c) shows a large bias towards the left of the zero line indicating that the air temperature in the plastic shield is significantly warmer than in the aluminum shield much of the time. Weekly mean differences exceed $0.15^{\circ} \mathrm{C}$.

The solar powered fan was intended to ventilate the shield during the day. However, the solar battery assembly was partially transparent, thus allowing solar energy to be transmitted and absorbed inside the shield. This raised the air temperature. In addition, the overall fan design may not be efficient for ventilating Thaller type shields. The air was probably not being drawn through the entire shield, but rather, through the top several plates just above the probe. Hence, it may not be feasible to ventilate a Thaller type shield in any simple manner.

Tables 19-22 categorize the mean differences into wind speed ranges for all data and for all daytime data. Again, the largest differences are found when winds are less than $2 \mathrm{~m} \mathrm{~s}^{-1}$. For these daytime data, the overall mean difference approaches $0.2^{\circ} \mathrm{C}$.

\subsection{Comparison of All Shields}

Results from tests of five different shields are examined and discussed in this section. The shields include an R. M. Young Gill multi-plate shield, a Met One and WHOI vane-oriented shield, and an R. M. Young Gill and Met One fan ventilated shield.

The Met One fan ventilated shield was used as a standard for comparison since its air temperature was the lowest. Scatter plots of air temperature difference as a function of insolation for a two week period are shown in Figures 9-12. Linear least square fits were 
computed for each scatter plot. Figure 9 shows the difference in air temperature between the R. M. Young Aspirated shield minus the Met One shield. For an insolation of $1000 \mathrm{Wm}^{-2}$, the linear fit error is $0.48^{\circ} \mathrm{C}$. The error for the R. M. Young Gill multi-plate shield is $0.51^{\circ} \mathrm{C}$ (Figure 10 ), $0.27^{\circ} \mathrm{C}$ for the Met One vane (Figure 11), and $0.43^{\circ} \mathrm{C}$ for the WHOI vane (Figure 12).

Probability density functions were constructed of air temperature difference for six weeks of data. Figure 13a displays the probability density of the R. M. Young Aspirated shield minus the Met One shield. The R. M. Young shield was inferior to the Met One shield (Figure 13c). An error of 0.1 to $0.2^{\circ} \mathrm{C}$ is most frequently observed but errors of more than $0.5^{\circ} \mathrm{C}$ are sometimes experienced. A prototype of a new R. M. Young shield is now being tested.

For the R. M. Young Gill multi-plate shield (Figure 14) a temperature error of 0.1 to $0.2^{\circ} \mathrm{C}$ is frequently observed with occasional errors in excess of $0.5^{\circ} \mathrm{C}$ due to large solar heating. Agreement at night is very good.

Both vane-oriented shields behave in a similar manner. The Met One vane (Figure 15) and WHOI vane (Figure 16) have errors usually less than $0.5^{\circ} \mathrm{C}$. The evening values show offsets to the left side of the graphs, indicating that both of these vanes are observing cooler air temperatures than the Met One shield. This is difficult to explain. One possibility is that heat may be generated by the fan on the Met One shield and is keeping the temperature in the shield slightly warmer or it may just be a bias in the recording system. The errors are evenly distributed on both sides of the zero line within $\pm 0.3^{\circ} \mathrm{C}$. Of the two vanes, the Met One vane minimizes the solar heating. The WHOI vane has larger errors (greater than $0.5^{\circ} \mathrm{C}$ ) more frequently.

\section{Summary and Conclusions}

Performance of radiation shields has been examined as part of the IMET project. Static and vane-oriented shields are being considered for use on buoys while fan ventilated shields are being evaluated for use on ships where power is readily available. These shields have been tested and the results are discussed. 
The mean difference of air temperatures inside two R. M. Young Gill multi-plate radiation shields is of order $0.01^{\circ} \mathrm{C}$. This difference is much less than the accuracy of the data acquisition system. Differences on the order of several tenths of a degree are occasionally observed when winds are light and solar heating large. Differential heating of the meteorology tower is assumed to be the contributing factor. Past studies (Gill, 1983; McKay and McTaggart-Cowan, 1977) have shown that, when compared to other static shields, the Gill multi-plate radiation shield has the best performance.

There is no significant difference between similar Thaller type shields made of plastic and aluminum. The overall mean air temperature difference is approximately $0.02^{\circ} \mathrm{C}$ which is about one order of magnitude smaller than the accuracy of the recording system. More variability in these data was observed than with two plastic multi-plate shields. It may be advantageous to use the plastic since the aluminum may corrode in the marine environment.

The top plate of a plastic Thaller shield was removed and replaced with a solar powered fan. Data suggest that this design did not work well as heat was transmitted through the top of the battery assembly into the shield. Also, the fan was probably not drawing air through the entire shield, but rather through the top several plates which are above the probe.

The Met One fan ventilated shield was used as a standard to compare the other shields against. An obsolete R. M. Young Aspirated shield was included for comparison purposes only and did not perform as well as the Met One shield.

A vane-oriented shield may be more desirable for use on buoys were power requirements are limited. The preliminary results presented here suggest that a vane may work better than a static shield because of the ability of the vane to orient itself into the wind. However, the mechanical design must be improved (e.g., minimize corrosion effects). Of the two vanes, the Met One shows a slightly better performance. More engineering modifications are anticipated on the WHOI vane. 


\section{Acknowledgements}

The authors wish to thank Jerome Dean for testing his vane oriented radiation shield. Thanks goes out to Ovid Forier for his help in designing and constructing the circuitry for the data acquisition system. The authors would also like to thank Barbara Gaffron for her helpful suggestions and comments.

This work was funded by the National Science Foundation (Grant OCE-8709614) as a World Ocean Circulation Experiment (WOCE) long-lead time development activity. 


\section{References}

Dean, J. P., and R. C. Beardsley, 1988. A vector-averaging wind recorder (VAWR) system for surface meteorological measurements in CODE (Coastal Ocean Dynamics Experiment). Woods Hole Oceanographic Institution Technical Report, WHOI-88-20, $74 \mathrm{pp}$.

Franssila, M., 1961. Radiation shield for microclimatological measuring of temperature. Geophysica, 8, 85-88.

Fuchs, M., and C. B. Tanner, 1965. Radiation shields for air temperature thermometers. Journal of Applied Meteorology, 4, 544-547.

Gill, G. C., 1979. Development of a small rugged radiation shield for air temperature measurements on drifting buoys. Report on research sponsored by the NOAA Data Buoy Office, Bay St. Louis, Mississippi, 23 pp., 17 figs.

Gill, G. C., 1983. Comparison testing of selected naturally ventilated solar radiation shields. Report submitted to NOAA Data Buoy Office, Bay St. Louis, Mississippi. In partial fulfillment of Contract \#NA-82-0A-A-266, September 1983.

Hadlock, R., W. R. Seguin, and M. Garstang, 1972. A radiation shield for thermistor deployment in the atmospheric boundary layer. Journal of Applied Meteorology, 11, 393-399.

MacHattie, L. B., 1965. Radiation screens for air temperature measurements. Ecology, 46, 533-538.

McKay, D. J., and J. D. McTaggart-Cowan, 1977. An intercomparison of radiation shields for auto stations. WMO Technical Conference on Instruments and Methods of Observation (TECIMO), Hamburg, WMO No. 480 , pp. 208-213.

Payne, R. E., 1974. A buoy-mounted meteorological recording package. Woods Hole Oceanographic Institution Technical Report, WHOI-74-40, 32 pp. 
Payne, R. E., 1987. Air temperature shield tests. Woods Hole Oceanographic Institution Technical Report, WHOI-87-40, 22 pp.

Payne, R. E., 1988. The MR: A meteorological data sensing, recording and telemetering package for use on moored buoys. Journal of Atmospheric and Oceanic Technology, $\mathbf{5}$, 286-297.

Payne, R. E., A. L. Bradshaw, J. P. Dean, and K. E. Schleicher, 1976. Accuracy of temperature measurements with the VACM. Woods Hole Oceanographic Institution Technical Report, WHOI-76-94, 78 pp.

Pech, G., 1964. A practical thermocouple shield. Forestry Chronicle, 40, 184-186.

Sparks, W. R., 1972. The effect of thermometer screen design on the observed temperature. World Meteorological Organization, WMO No. 315, 106 pp. 


\section{Appendix A - Manufacturer Addresses}

Met One, Inc

481 California Avenue

Grants Pass, Oregon 97526

(503) 479-1248

Vane Aspirated Temperature Shield (071A)

$\$ 430$

Air Temperature Aspirated Radiation Shield (076)

R. M. Young Company

2801 Aero-Park Drive

Traverse City, Michigan 49684

(616) 946-3980

Gill Multi-Plate Radiation Shield (41002)

Gill Aspirated Radiation Shield (43404)

Yellow Springs Instrument Company, Inc.

Yellow Springs, Ohio 45387

(513) 767-7241

Precision Thermistor (44032)

$\$ 16.20$ 
Table 1: Manufacturer Specifications for Radiation Shields

$\begin{array}{lcccc}\text { Manufacturer } & \text { Young } & \text { Young } & \text { Met One } & \text { Met One } \\ \text { Model } & 41002 & 43404 & 076 & 071 \mathrm{~A} \\ \begin{array}{l}\text { Temperature } \\ \text { Error (C) }\end{array} & \begin{array}{l}1.5 \text { @ } 1 \mathrm{~m} \mathrm{~s}^{-1} \\ 0.4 @ 2 \mathrm{~m} \mathrm{~s}^{-1}\end{array} & 0.2 & 0.03 & 0.1 \\ & 0.3 \mathrm{~m} \mathrm{~s}^{-1} & & & \text { @ }>1.5 \mathrm{~m} \mathrm{~s}^{-1} \\ \text { Maximum Solar } \\ \begin{array}{l}\text { Intensity }\left(\mathrm{Wm}^{-2}\right) \\ \text { Aspiration }\left(\mathrm{m} \mathrm{s}^{-1}\right)\end{array} & 1080 & 1080 & 840 & 1120 \\ \text { variable } & 3.0 & 2.5 & \text { variable } \\ \text { Cost } & \$ 158 & - & \$ 697 & \$ 430\end{array}$


Table 2: Comparison of Two Identical Plastic Gill Multi-Plate Shields

All Data

\begin{tabular}{|c|c|c|c|c|c|c|c|}
\hline \multirow[b]{2}{*}{$\begin{array}{c}\text { Date } \\
\text { (YYWK) }\end{array}$} & \multirow[b]{2}{*}{ Recs } & \multicolumn{3}{|c|}{ Mean } & \multicolumn{3}{|c|}{ Standard Deviation } \\
\hline & & $\begin{array}{c}\text { YSI-10 } \\
(\mathrm{C})\end{array}$ & $\begin{array}{c}\text { YSI-20 } \\
(\mathrm{C})\end{array}$ & $\begin{array}{c}10-20 \\
(\mathrm{C})\end{array}$ & $\begin{array}{c}\text { YSI-10 } \\
\text { (C) }\end{array}$ & $\begin{array}{c}\text { YSI-20 } \\
(\mathrm{C})\end{array}$ & $\begin{array}{r}10-20 \\
(\mathrm{C}) \\
\end{array}$ \\
\hline 8825 & 271 & 15.395 & 15.393 & 0.002 & 1.282 & 1.276 & 0.060 \\
\hline 8826 & 622 & 16.672 & 16.690 & -0.018 & 1.722 & 1.738 & 0.092 \\
\hline 8827 & 476 & 19.443 & 19.477 & -0.034 & 1.610 & 1.606 & 0.158 \\
\hline 8828 & 1174 & 21.065 & 21.063 & 0.003 & 1.644 & 1.647 & 0.077 \\
\hline 8829 & 1342 & 21.397 & 21.414 & -0.017 & 1.637 & 1.654 & 0.077 \\
\hline 8830 & 1342 & 21.582 & 21.587 & -0.005 & 1.476 & 1.473 & 0.055 \\
\hline 8831 & 1339 & 23.235 & 23.254 & -0.019 & 1.281 & 1.279 & 0.091 \\
\hline 8832 & 1342 & 23.689 & 23.690 & -0.001 & 0.999 & 1.004 & 0.049 \\
\hline 8833 & 1341 & 21.472 & 21.488 & -0.016 & 1.985 & 1.983 & 0.058 \\
\hline 8834 & 1338 & 18.667 & 18.692 & -0.024 & 2.366 & 2.372 & 0.093 \\
\hline 8835 & 1342 & 20.303 & 20.324 & -0.021 & 2.123 & 2.128 & 0.070 \\
\hline 8836 & 1343 & 18.506 & 18.526 & -0.019 & 2.186 & 2.172 & 0.103 \\
\hline 8837 & 1344 & 16.732 & 16.764 & -0.032 & 2.317 & 2.296 & 0.094 \\
\hline 8838 & 487 & 18.355 & 18.377 & -0.022 & 1.446 & 1.470 & 0.093 \\
\hline 8844 & 450 & 11.738 & 11.752 & -0.013 & 2.141 & 2.155 & 0.059 \\
\hline 8845 & 1343 & 10.196 & 10.209 & -0.013 & 3.077 & 3.081 & 0.053 \\
\hline 8846 & 1344 & 9.345 & 9.358 & -0.013 & 3.052 & 3.052 & 0.051 \\
\hline 8847 & 1344 & 6.021 & 6.038 & -0.017 & 3.643 & 3.635 & 0.075 \\
\hline 8848 & 1344 & 7.934 & 7.954 & -0.020 & 3.414 & 3.411 & 0.060 \\
\hline 8849 & 1341 & 2.851 & 2.868 & -0.017 & 4.530 & 4.536 & 0.047 \\
\hline 8850 & 1344 & -2.361 & -2.357 & -0.004 & 6.029 & 6.035 & 0.058 \\
\hline 8851 & 1343 & 2.511 & 2.522 & -0.011 & 4.668 & 4.676 & 0.054 \\
\hline 8852 & 1343 & 2.912 & 2.924 & -0.012 & 3.867 & 3.859 & 0.076 \\
\hline 8901 & 1344 & -2.379 & -2.360 & -0.019 & 5.134 & 5.135 & 0.056 \\
\hline 8902 & 1343 & 1.920 & 1.937 & -0.017 & 3.193 & 3.193 & 0.051 \\
\hline 8903 & 1344 & 2.794 & 2.801 & -0.007 & 3.421 & 3.402 & 0.090 \\
\hline 8904 & 1344 & 1.637 & 1.657 & -0.020 & 3.558 & 3.558 & 0.071 \\
\hline 8905 & 1344 & 3.102 & 3.111 & -0.009 & 3.567 & 3.579 & 0.059 \\
\hline 8906 & 1344 & -3.270 & -3.272 & 0.002 & 3.195 & 3.201 & 0.060 \\
\hline 8907 & 1344 & -0.073 & -0.069 & -0.004 & 4.575 & 4.586 & 0.064 \\
\hline 8908 & 1344 & 1.115 & 1.102 & 0.013 & 4.598 & 4.607 & 0.054 \\
\hline 8909 & 1344 & -0.300 & -0.290 & -0.011 & 2.156 & 2.167 & 0.074 \\
\hline 8910 & 1344 & -1.863 & -1.877 & 0.014 & 3.262 & 3.259 & 0.092 \\
\hline 8911 & 1344 & 3.384 & 3.390 & -0.006 & 3.129 & 3.143 & 0.092 \\
\hline 8912 & 885 & 1.657 & 1.652 & 0.004 & 2.241 & 2.257 & 0.122 \\
\hline Total & 41967 & 8.917 & 8.929 & -0.012 & 3.252 & 3.254 & 0.074 \\
\hline
\end{tabular}


Table 3: Comparison of Two Identical Plastic Gill Multi-Plate Shields Nighttime Data (less than $5 \mathrm{Wm}^{-2}$ )

\begin{tabular}{|c|c|c|c|c|c|c|c|}
\hline \multirow[b]{2}{*}{$\begin{array}{c}\text { Date } \\
(\mathrm{YYWK})\end{array}$} & \multirow[b]{2}{*}{ Recs } & \multicolumn{3}{|c|}{ Mean } & \multicolumn{3}{|c|}{ Standard Deviation } \\
\hline & & $\begin{array}{c}\text { YSI-10 } \\
(\mathrm{C})\end{array}$ & $\begin{array}{c}\text { YSI-20 } \\
(\mathrm{C})\end{array}$ & $\begin{array}{c}10-20 \\
(\mathrm{C}) \\
\end{array}$ & $\begin{array}{c}\text { YSI-10 } \\
(\mathrm{C})\end{array}$ & $\begin{array}{c}\text { YSI-20 } \\
(\mathrm{C})\end{array}$ & $\begin{array}{c}10-20 \\
(\mathrm{C}) \\
\end{array}$ \\
\hline 8825 & 73 & 13.811 & 13.809 & 0.002 & 0.163 & 0.162 & 0.015 \\
\hline 8826 & 242 & 15.654 & 15.651 & 0.003 & 1.195 & 1.203 & 0.028 \\
\hline 8827 & 163 & 18.536 & 18.530 & 0.006 & 1.315 & 1.320 & 0.026 \\
\hline 8828 & 460 & 19.827 & 19.817 & 0.010 & 0.751 & 0.753 & 0.032 \\
\hline 8828 & 549 & 20.815 & 20.821 & -0.006 & 1.332 & 1.326 & 0.041 \\
\hline 8830 & 550 & 20.733 & 20.733 & 0.001 & 1.052 & 1.039 & 0.036 \\
\hline 8831 & 549 & 22.463 & 22.465 & -0.002 & 0.895 & 0.893 & 0.027 \\
\hline 8832 & 568 & 23.097 & 23.091 & 0.005 & 0.660 & 0.659 & 0.025 \\
\hline 8833 & 586 & 21.097 & 21.091 & 0.006 & 2.227 & 2.227 & 0.028 \\
\hline 8834 & 602 & 17.782 & 17.775 & 0.007 & 1.886 & 1.891 & 0.027 \\
\hline 8835 & 624 & 19.639 & 19.637 & 0.002 & 2.060 & 2.062 & 0.013 \\
\hline 8836 & 645 & 17.974 & 17.977 & -0.003 & 1.937 & 1.934 & 0.082 \\
\hline 8837 & 655 & 16.542 & 16.538 & 0.004 & 2.398 & 2.395 & 0.032 \\
\hline 8838 & 277 & 17.695 & 17.696 & -0.001 & 0.559 & 0.555 & 0.042 \\
\hline 8844 & 247 & 11.578 & 11.596 & -0.018 & 2.299 & 2.292 & 0.022 \\
\hline 8845 & 787 & 10.165 & 10.181 & -0.016 & 3.149 & 3.150 & 0.031 \\
\hline 8846 & 806 & 9.136 & 9.153 & -0.018 & 3.091 & 3.091 & 0.036 \\
\hline 8847 & 820 & 5.675 & 5.693 & -0.018 & 4.068 & 4.064 & 0.039 \\
\hline 8848 & 839 & 7.636 & 7.651 & -0.014 & 3.366 & 3.365 & 0.048 \\
\hline 8849 & 825 & 3.066 & 3.084 & -0.019 & 4.575 & 4.577 & 0.020 \\
\hline 8850 & 835 & -2.652 & -2.649 & -0.003 & 6.209 & 6.212 & 0.050 \\
\hline 8851 & 843 & 2.282 & 2.294 & -0.012 & 4.847 & 4.857 & 0.040 \\
\hline 8852 & 838 & 2.966 & 2.974 & -0.008 & 4.115 & 4.108 & 0.073 \\
\hline 8901 & 833 & -2.627 & -2.619 & -0.008 & 5.243 & 5.245 & 0.040 \\
\hline 8902 & 831 & 1.663 & 1.679 & -0.016 & 3.391 & 3.392 & 0.039 \\
\hline 8903 & 812 & 2.763 & 2.776 & -0.013 & 2.646 & 2.631 & 0.055 \\
\hline 8904 & 804 & 0.847 & 0.862 & -0.015 & 3.853 & 3.856 & 0.060 \\
\hline 8905 & 796 & 3.163 & 3.170 & -0.007 & 3.268 & 3.276 & 0.056 \\
\hline 8906 & 775 & -3.716 & -3.714 & -0.002 & 3.115 & 3.122 & 0.045 \\
\hline 8907 & 762 & -0.124 & -0.118 & -0.006 & 4.099 & 4.099 & 0.037 \\
\hline 8908 & 759 & 0.925 & 0.915 & 0.010 & 4.174 & 4.177 & 0.045 \\
\hline 8909 & 720 & -0.690 & -0.680 & -0.010 & 2.084 & 2.085 & 0.046 \\
\hline 8910 & 709 & -2.160 & -2.157 & -0.003 & 2.911 & 2.905 & 0.050 \\
\hline 8911 & 692 & 2.726 & 2.740 & -0.014 & 3.036 & 3.039 & 0.052 \\
\hline 8912 & 442 & 1.236 & 1.252 & -0.016 & 2.029 & 2.029 & 0.059 \\
\hline Total & 22318 & 7.377 & 7.384 & -0.007 & 3.344 & 3.345 & 0.045 \\
\hline
\end{tabular}


Table 4: Comparison of Two Identical Plastic Gill Multi-Plate Shields Daytime Data (Greater than $5 \mathrm{Wm}^{-2}$ )

\begin{tabular}{|c|c|c|c|c|c|c|c|}
\hline \multirow[b]{2}{*}{$\begin{array}{c}\text { Date } \\
(\mathrm{YYWK})\end{array}$} & \multirow[b]{2}{*}{ Recs } & \multicolumn{3}{|c|}{ Mean } & \multicolumn{3}{|c|}{ Standard Deviation } \\
\hline & & $\begin{array}{c}\text { YSI-10 } \\
\text { (C) }\end{array}$ & $\begin{array}{c}\text { YSI-20 } \\
\text { (C) }\end{array}$ & $\begin{array}{c}10-20 \\
(\mathrm{C})\end{array}$ & $\begin{array}{l}\text { YSI-10 } \\
(\mathrm{C})\end{array}$ & $\begin{array}{c}\text { YSI-20 } \\
\text { (C) }\end{array}$ & $\begin{array}{c}10-20 \\
\text { (C) }\end{array}$ \\
\hline 8825 & 198 & 15.979 & 5.977 & 0.002 & 0.985 & 0.975 & 0.069 \\
\hline 8826 & 380 & 17.321 & 17.352 & -0.032 & 1.693 & 1.703 & 0.114 \\
\hline 8827 & 313 & 19.916 & 19.971 & -0.055 & 1.547 & 1.520 & 0.191 \\
\hline 8828 & 714 & 21.863 & 21.865 & -0.002 & 1.568 & 1.567 & 0.095 \\
\hline 8828 & 793 & 21.801 & 21.825 & -0.024 & 1.707 & 1.733 & 0.094 \\
\hline 8830 & 792 & 22.171 & 22.180 & -0.009 & 1.441 & 1.439 & 0.065 \\
\hline 8831 & 790 & 23.771 & 23.802 & -0.031 & 1.235 & 1.219 & 0.115 \\
\hline 8832 & 774 & 24.123 & 24.128 & -0.005 & 0.982 & 0.988 & 0.061 \\
\hline 8833 & 755 & 21.763 & 21.797 & -0.034 & 1.721 & 1.710 & 0.068 \\
\hline 8834 & 736 & 19.392 & 19.441 & -0.050 & 2.471 & 2.462 & 0.117 \\
\hline 8835 & 718 & 20.880 & 20.922 & -0.041 & 2.006 & 2.002 & 0.090 \\
\hline 8836 & 698 & 18.998 & 19.032 & -0.035 & 2.287 & 2.257 & 0.117 \\
\hline 8837 & 689 & 16.913 & 16.979 & -0.066 & 2.223 & 2.177 & 0.118 \\
\hline 8838 & 210 & 19.226 & 19.275 & -0.050 & 1.763 & 1.786 & 0.127 \\
\hline 8844 & 203 & 11.934 & 11.941 & -0.008 & 1.920 & 1.964 & 0.085 \\
\hline 8845 & 556 & 10.239 & 10.248 & -0.009 & 2.975 & 2.983 & 0.073 \\
\hline 8846 & 538 & 9.659 & 9.665 & -0.006 & 2.966 & 2.968 & 0.067 \\
\hline 8847 & 524 & 6.561 & 6.577 & -0.016 & 2.772 & 2.756 & 0.110 \\
\hline 8848 & 505 & 8.429 & 8.459 & -0.030 & 3.440 & 3.429 & 0.076 \\
\hline 8849 & 516 & 2.507 & 2.522 & -0.015 & 4.439 & 4.453 & 0.072 \\
\hline 8850 & 509 & -1.884 & -1.877 & -0.007 & 5.695 & 5.705 & 0.070 \\
\hline 8851 & 500 & 2.895 & 2.905 & -0.010 & 4.326 & 4.331 & 0.071 \\
\hline 8852 & 505 & 2.821 & 2.841 & -0.020 & 3.417 & 3.408 & 0.080 \\
\hline 8901 & 511 & -1.974 & -1.937 & -0.037 & 4.930 & 4.927 & 0.073 \\
\hline 8902 & 512 & 2.338 & 2.356 & -0.018 & 2.795 & 2.795 & 0.066 \\
\hline 8903 & 532 & 2.841 & 2.840 & 0.001 & 4.348 & 4.323 & 0.126 \\
\hline 8904 & 540 & 2.813 & 2.840 & -0.027 & 2.668 & 2.653 & 0.085 \\
\hline 8905 & 548 & 3.014 & 3.026 & -0.012 & 3.962 & 3.981 & 0.063 \\
\hline 8906 & 569 & -2.663 & -2.670 & 0.008 & 3.205 & 3.213 & 0.076 \\
\hline 8907 & 582 & -0.007 & -0.006 & -0.001 & 5.134 & 5.157 & 0.087 \\
\hline 8908 & 585 & 1.362 & 1.345 & 0.018 & 5.088 & 5.105 & 0.065 \\
\hline 8909 & 624 & 0.150 & 0.162 & -0.012 & 2.152 & 2.173 & 0.097 \\
\hline 8910 & 635 & -1.531 & -1.564 & 0.033 & 3.588 & 3.590 & 0.120 \\
\hline 8911 & 652 & 4.082 & 4.080 & 0.002 & 3.076 & 3.106 & 0.120 \\
\hline 8912 & 443 & 2.027 & 2.052 & 0.025 & 2.362 & 2.401 & 0.159 \\
\hline Total & 19649 & 10.666 & 10.683 & -0.017 & 3.060 & 3.062 & 0.096 \\
\hline
\end{tabular}


Table 5: Mean Differences of Two Identical Plastic

Gill Multi-Plate Shields by Wind Speed

(YSI-10-YSI-20) All Data

\begin{tabular}{|c|c|c|c|c|c|c|c|c|c|}
\hline $\begin{array}{c}\text { Date } \\
\text { (YYWK) }\end{array}$ & $\begin{array}{r}0-1 \\
\mathrm{~m} \mathrm{~s}^{-1} \\
\end{array}$ & $\begin{array}{r}1-2 \\
\mathrm{~m} \mathrm{~s}^{-1} \\
\end{array}$ & $\begin{array}{r}2-3 \\
\mathrm{~m} \mathrm{~s}^{-1} \\
\end{array}$ & $\begin{array}{r}3-4 \\
\mathrm{~m} \mathrm{~s}^{-1} \\
\end{array}$ & $\begin{array}{r}4-5 \\
\mathrm{~m} \mathrm{~s}^{-1} \\
\end{array}$ & $\begin{array}{r}5-6 \\
\mathrm{~m} \mathrm{~s}^{-1} \\
\end{array}$ & $\begin{array}{r}6-7 \\
\mathrm{~m} \mathrm{~s}^{-1} \\
\end{array}$ & $\begin{array}{r}7-8 \\
\mathrm{~m} \mathrm{~s}^{-1} \\
\end{array}$ & $\begin{array}{r}>8 \\
\mathrm{~m} \mathrm{~s}^{-1}\end{array}$ \\
\hline 8825 & -0.002 & -0.006 & -0.012 & -0.007 & 0.007 & 0.037 & 0.017 & 0.010 & 0.013 \\
\hline 8826 & -0.200 & -0.071 & -0.026 & 0.017 & -0.008 & -0.010 & -0.013 & -0.003 & -0.011 \\
\hline 8827 & -0.309 & -0.106 & -0.109 & -0.037 & 0.003 & -0.007 & -0.055 & -0.013 & 0.025 \\
\hline 8828 & 0.063 & 0.002 & 0.014 & 0.022 & -0.016 & -0.002 & -0.012 & 0.002 & -0.000 \\
\hline 8829 & -0.039 & -0.068 & -0.033 & -0.004 & -0.003 & 0.003 & -0.008 & 0.008 & 0.008 \\
\hline 8830 & -0.057 & -0.021 & -0.008 & -0.001 & 0.007 & 0.005 & 0.003 & -0.002 & 0.005 \\
\hline 8831 & -0.025 & -0.032 & -0.025 & -0.036 & -0.029 & -0.023 & -0.008 & 0.005 & 0.012 \\
\hline 8832 & -0.138 & -0.076 & -0.020 & -0.000 & 0.002 & 0.003 & 0.004 & 0.012 & 0.013 \\
\hline 8833 & -0.066 & -0.032 & -0.023 & -0.028 & -0.012 & -0.013 & -0.020 & -0.009 & 0.002 \\
\hline 8834 & -0.045 & -0.040 & -0.048 & -0.023 & -0.011 & 0.000 & 0.008 & -0.001 & 0.019 \\
\hline 8835 & -0.005 & -0.027 & -0.040 & -0.025 & -0.030 & -0.011 & -0.001 & -0.005 & 0.005 \\
\hline 8836 & 0.012 & -0.082 & -0.030 & -0.011 & -0.012 & -0.027 & -0.038 & -0.005 & 0.006 \\
\hline 8837 & 051 & -0.095 & -0.051 & -0.074 & -0.0 & -0.011 & 0.006 & 00 & -0.005 \\
\hline 8838 & -0.002 & -0.079 & -0.021 & -0.018 & -0.013 & -0.005 & -0.006 & -0.013 & 0.001 \\
\hline 8844 & 0.029 & 0.074 & -0.018 & -0.043 & -0.033 & -0.023 & -0.010 & -0.007 & 0.023 \\
\hline 8845 & -0.005 & 007 & 0.009 & -0.013 & -0.012 & -0.021 & -0.012 & -0.032 & -0.018 \\
\hline 8846 & -0.032 & -0.004 & 0.008 & 0.003 & -0.029 & -0.012 & -0.010 & -0.016 & -0.025 \\
\hline 8847 & -0.059 & -0.045 & -0.003 & -0.022 & -0.017 & -0.013 & -0.003 & 0.020 & -0.010 \\
\hline 8848 & -0.060 & -0.087 & -0.051 & -0.008 & -0.006 & -0.013 & -0.021 & -0.028 & -0.016 \\
\hline 8849 & - & 0.015 & -0.001 & -0.012 & -0.018 & -0.022 & -0.009 & -0.014 & -0.027 \\
\hline 8850 & 31 & 0.013 & 0.019 & 0.007 & 0.004 & -0. & -0.012 & & -0.008 \\
\hline 8851 & - & -0.007 & 0.011 & -0.018 & -0.022 & -0.011 & -0.014 & -0.003 & -0.011 \\
\hline 8852 & - & - & 0.051 & -0.006 & -0.009 & -0.020 & -0.007 & -0.025 & -0.011 \\
\hline 8901 & - & - & -0.023 & -0.019 & -0.041 & -0.015 & -0.013 & -0.009 & -0.023 \\
\hline 8902 & - & -0.005 & -0.005 & 0.005 & -0.007 & -0.011 & -0.016 & -0.015 & -0.023 \\
\hline 8903 & - & -0.044 & -0.027 & -0.006 & -0.003 & -0.009 & -0.029 & -0.027 & 0.005 \\
\hline 8904 & -0.028 & -0.037 & -0.012 & -0.017 & -0.013 & -0.014 & -0.020 & -0.018 & -0.022 \\
\hline 8905 & -0.002 & -0.007 & -0.005 & -0.001 & -0.009 & -0.007 & -0.015 & -0.017 & -0.014 \\
\hline 8906 & -0.000 & 0.008 & 0.008 & 0.008 & 0.008 & 0.003 & -0.001 & 0.006 & -0.006 \\
\hline 8907 & - & -0.018 & -0.002 & -0.001 & -0.014 & 0.006 & 0.009 & -0.002 & -0.022 \\
\hline 8908 & 0.022 & -0.002 & -0.007 & 0.017 & 0.031 & 0.015 & 0.006 & 0.008 & 0.013 \\
\hline 8909 & 0.010 & -0.026 & -0.014 & -0.020 & -0.013 & 0.004 & 0.002 & -0.007 & -0.009 \\
\hline 8910 & 0.019 & 0.020 & 0.272 & 0.100 & 0.073 & 0.022 & 0.013 & 0.020 & 0.007 \\
\hline 8911 & 0.010 & 0.014 & -0.022 & -0.012 & 0.005 & 0.005 & -0.001 & -0.010 & -0.007 \\
\hline 8912 & - & 0.210 & 0.062 & 0.025 & 0.012 & 0.008 & 0.000 & -0.009 & 0.002 \\
\hline Total & -0.033 & -0.032 & -0.018 & -0.013 & -0.010 & -0.007 & -0.008 & -0.007 & -0.008 \\
\hline
\end{tabular}


Table 6: Wind Speed Distribution

\section{All Data}

\begin{tabular}{|c|c|c|c|c|c|c|c|c|c|c|}
\hline $\begin{array}{c}\text { Date } \\
\text { (YYWK) }\end{array}$ & $\begin{array}{r}0-1 \\
\mathrm{~m} \mathrm{~s}^{-1}\end{array}$ & $\begin{array}{r}1-2 \\
\mathrm{~m} \mathrm{~s}^{-1}\end{array}$ & $\begin{array}{r}2-3 \\
\mathrm{~m} \mathrm{~s}^{-1}\end{array}$ & $\begin{array}{r}3-4 \\
\mathrm{~m} \mathrm{~s}^{-1}\end{array}$ & $\begin{array}{r}4-5 \\
\mathrm{~m} \mathrm{~s}^{-1}\end{array}$ & $\begin{array}{r}5-6 \\
\mathrm{~m} \mathrm{~s}^{-1}\end{array}$ & $\begin{array}{r}6-7 \\
\mathrm{~m} \mathrm{~s}^{-1}\end{array}$ & $\begin{array}{r}7-8 \\
\mathrm{~m} \mathrm{~s}^{-1}\end{array}$ & $\begin{array}{r}>8 \\
\mathrm{~m} \mathrm{~s}^{-1}\end{array}$ & Total \\
\hline 8825 & 11 & 57 & 33 & 65 & 50 & 26 & 14 & 10 & 5 & 271 \\
\hline 8826 & 6 & 90 & 124 & 126 & 86 & 63 & 63 & 48 & 16 & 622 \\
\hline 8827 & 4 & 35 & 51 & 52 & 84 & 110 & 63 & 44 & 33 & 476 \\
\hline 8828 & 19 & 122 & 182 & 170 & 196 & 194 & 133 & 104 & 54 & 1174 \\
\hline 8829 & 7 & 188 & 257 & 238 & 205 & 165 & 107 & 84 & 91 & 1342 \\
\hline 8830 & 47 & 188 & 277 & 253 & 187 & 168 & 127 & 73 & 22 & 1342 \\
\hline 8831 & 59 & 173 & 183 & 148 & 185 & 205 & 120 & 101 & 165 & 1339 \\
\hline 8832 & 2 & 61 & 90 & 169 & 217 & 265 & 233 & 145 & 160 & 1342 \\
\hline 8833 & 29 & 143 & 164 & 203 & 150 & 131 & 129 & 68 & 324 & 1341 \\
\hline 8834 & 42 & 218 & 306 & 292 & 170 & 132 & 85 & 50 & 43 & 1338 \\
\hline 8835 & 12 & 193 & 270 & 244 & 171 & 111 & 145 & 91 & 105 & 1342 \\
\hline 8836 & 9 & 93 & 213 & 238 & 175 & 136 & 122 & 111 & 246 & 1343 \\
\hline 8837 & 5 & 25 & 103 & 264 & 338 & 264 & 129 & 58 & 158 & 1344 \\
\hline 8838 & 7 & 74 & 68 & 105 & 39 & 57 & 46 & 43 & 48 & 487 \\
\hline 8844 & 8 & 25 & 37 & 66 & 109 & 77 & 47 & 22 & 59 & 450 \\
\hline 8845 & 12 & 91 & 112 & 135 & 212 & 196 & 158 & 99 & 328 & 1343 \\
\hline 8846 & 21 & 122 & 118 & 144 & 130 & 182 & 170 & 160 & 297 & 1344 \\
\hline 8847 & 51 & 142 & 111 & 238 & 285 & 167 & 74 & 56 & 220 & 1344 \\
\hline 8848 & 1 & 33 & 53 & 83 & 85 & 152 & 190 & 200 & 547 & 1344 \\
\hline 8849 & 0 & 21 & 122 & 151 & 160 & 191 & 149 & 99 & 448 & 1341 \\
\hline 8850 & 3 & 24 & 74 & 107 & 143 & 191 & 197 & 165 & 440 & 1344 \\
\hline 8851 & 0 & 9 & 45 & 91 & 102 & 177 & 182 & 181 & 556 & 1343 \\
\hline 8852 & 0 & 0 & 12 & 77 & 150 & 182 & 181 & 152 & 589 & 1343 \\
\hline 8901 & 0 & 0 & 5 & 8 & 31 & 83 & 159 & 249 & 809 & 1344 \\
\hline 8902 & 0 & 3 & 13 & 57 & 78 & 147 & 227 & 189 & 629 & 1343 \\
\hline 8903 & 0 & 2 & 6 & 47 & 114 & 177 & 217 & 151 & 630 & 1344 \\
\hline 8904 & 39 & 227 & 272 & 196 & 176 & 114 & 99 & 64 & 157 & 1344 \\
\hline 8905 & 1 & 92 & 126 & 191 & 253 & 218 & 172 & 106 & 185 & 1344 \\
\hline 8906 & 18 & 122 & 134 & 146 & 138 & 135 & 109 & 66 & 476 & 1344 \\
\hline 8907 & 0 & 2 & 20 & 109 & 143 & 257 & 286 & 232 & 295 & 1344 \\
\hline 8908 & 1 & 28 & 46 & 115 & 151 & 154 & 184 & 186 & 479 & 1344 \\
\hline 8909 & 2 & 59 & 135 & 223 & 158 & 91 & 70 & 102 & 504 & 1344 \\
\hline 8910 & 1 & 2 & 3 & 21 & 38 & 85 & 132 & 163 & 899 & 1344 \\
\hline 8911 & 5 & 63 & 212 & 164 & 139 & 120 & 143 & 101 & 397 & 1344 \\
\hline 8912 & 0 & 1 & 14 & 54 & 100 & 141 & 124 & 140 & 311 & 885 \\
\hline Total & 422 & 2728 & 3991 & 4990 & 5148 & 5264 & 4786 & 3913 & 10725 & 41967 \\
\hline
\end{tabular}


Table 7: Mean Differences of Two Identical Plastic

Gill Multi-Plate Shields by Wind Speed

(YSI-10-YSI-20) Daytime Data (Greater than $5 \mathrm{Wm}^{-2}$ )

\begin{tabular}{|c|c|c|c|c|c|c|c|c|c|}
\hline $\begin{array}{c}\text { Date } \\
(\text { YYWK })\end{array}$ & $\begin{array}{r}0-1 \\
\mathrm{~m} \mathrm{~s}^{-1} \\
\end{array}$ & $\begin{array}{r}1-2 \\
\mathrm{~m} \mathrm{~s}^{-1} \\
\end{array}$ & $\begin{array}{r}2-3 \\
\mathrm{~m} \mathrm{~s}^{-1} \\
\end{array}$ & $\begin{array}{r}3-4 \\
\mathrm{~m} \mathrm{~s}^{-1} \\
\end{array}$ & $\begin{array}{r}4-5 \\
\mathrm{~m} \mathrm{~s}^{-1} \\
\end{array}$ & $\begin{array}{r}5-6 \\
\mathrm{~m} \mathrm{~s} \mathrm{~s}^{-1} \\
\end{array}$ & $\begin{array}{r}6-7 \\
\mathrm{~m} \mathrm{~s}^{-1} \\
\end{array}$ & $\begin{array}{r}7-8 \\
\mathrm{~m} \mathrm{~s}^{-1} \\
\end{array}$ & $\begin{array}{r}>8 \\
\mathrm{~m} \mathrm{~s}^{-1} \\
\end{array}$ \\
\hline 8825 & -0.002 & -0.0 & -0.028 & -0.013 & 0.011 & 0.037 & 0.017 & 0.010 & 0.013 \\
\hline 8826 & -0.241 & -0.125 & -0.045 & 0.033 & -0.015 & -0.021 & -0.019 & -0.005 & -0.011 \\
\hline 8827 & -0.309 & -0.219 & -0.236 & -0.064 & 0.005 & -0.015 & -0.062 & -0.013 & 0.025 \\
\hline 8828 & 0.134 & -0.023 & 0.018 & 0.027 & -0.029 & -0.008 & -0.021 & 0.001 & -0.000 \\
\hline 8829 & -0.159 & -0.146 & -0.044 & -0.004 & -0.004 & 0.006 & -0.012 & 0.025 & 0.016 \\
\hline 8830 & -0.097 & -0.038 & -0.018 & 0.001 & 0.006 & 0.006 & -0.001 & -0.008 & 0.002 \\
\hline 8831 & -0.046 & -0.057 & -0.039 & -0.077 & -0.076 & -0.042 & -0.012 & 0.007 & 0.012 \\
\hline 8832 & -0.138 & -0.101 & -0.052 & -0.010 & 0.004 & 0.001 & 0.002 & 0.008 & 0.013 \\
\hline 8833 & -0.105 & -0.057 & -0.052 & -0.062 & -0.027 & -0.026 & -0.036 & -0.022 & -0.000 \\
\hline 8834 & -0.065 & -0.101 & -0.109 & -0.040 & -0.028 & -0.005 & 0.010 & 0.001 & 0.019 \\
\hline 8835 & 0.002 & -0.073 & -0.083 & -0.043 & -0.040 & -0.015 & -0.003 & -0.013 & 0.004 \\
\hline 8836 & 0.040 & -0.148 & -0.050 & -0.017 & -0.031 & -0.050 & -0.065 & -0.004 & -0.001 \\
\hline 8837 & 0.051 & -0.095 & -0.079 & -0.129 & -0.079 & -0.038 & -0.003 & -0.004 & -0.008 \\
\hline 8838 & -0.002 & -0.126 & -0.063 & -0.071 & -0.023 & -0.005 & 0.004 & -0.015 & 0.001 \\
\hline 8844 & 0.126 & 0.195 & -0.030 & -0.065 & -0.049 & -0.028 & -0.001 & 0.008 & 0.037 \\
\hline 8845 & 0.112 & 0.035 & 0.033 & -0.004 & -0.017 & -0.024 & -0.002 & -0.037 & -0.023 \\
\hline 8846 & 0.025 & 0.022 & 0.046 & 0.027 & -0.037 & 0.001 & 0.011 & -0.018 & -0.036 \\
\hline 8847 & -0.131 & -0.113 & 0.007 & -0.031 & -0.013 & -0.008 & 0.018 & 0.052 & -0.013 \\
\hline 8848 & -0.060 & -0.151 & -0.095 & 0.009 & 0.004 & -0.002 & -0.022 & -0.029 & -0.030 \\
\hline 8849 & - & 0.123 & 0.035 & 0.001 & -0.022 & -0.032 & 0. & -0 . & -0.044 \\
\hline 8850 & - & - & -0.000 & -0.006 & 0.015 & -0.000 & -0.010 & -0.016 & -0.015 \\
\hline 8851 & - & -0.009 & 0.046 & 0.003 & -0.036 & -0.018 & -0.013 & 0.003 & -0.013 \\
\hline 8852 & - & - & 0.077 & -0.009 & -0.004 & -0.028 & -0.019 & -0.040 & -0.023 \\
\hline 8901 & - & - & -0.024 & -0.017 & -0.042 & -0.029 & -0.024 & -0.027 & -0.045 \\
\hline 8902 & - & 0.001 & 0.036 & 0.019 & 0.008 & -0.000 & -0.013 & -0.011 & -0.032 \\
\hline 8903 & - & - & -0.015 & 0.006 & 0.013 & -0.000 & -0.033 & -0.038 & 0.022 \\
\hline 8904 & -0.047 & -0.068 & -0.016 & -0.023 & -0.018 & -0.011 & -0.020 & -0.025 & -0.024 \\
\hline 8905 & - & 0.001 & -0.001 & 0.008 & -0.008 & -0.009 & -0.015 & -0.023 & -0.044 \\
\hline 8906 & 0.004 & 0.010 & -0.008 & 0.020 & 0.023 & 0.023 & 0.015 & 0.034 & -0.003 \\
\hline 8907 & - & - & 0.011 & 0.010 & -0.021 & 0.015 & 0.024 & 0.007 & -0.038 \\
\hline 8908 & 0.022 & -0.000 & -0.000 & 0.021 & 0.016 & 0.017 & 0.026 & 0.028 & 0.016 \\
\hline 8909 & 0.050 & -0.066 & -0.024 & -0.023 & -0.035 & 0.044 & 0.009 & 0.001 & -0.007 \\
\hline 8910 & 0.019 & 0.020 & 0.272 & 0.114 & 0.116 & 0.076 & 0.048 & 0.051 & 0.016 \\
\hline 8911 & - & 0.065 & -0.032 & -0.008 & 0.033 & 0.032 & 0.001 & -0.010 & 0.000 \\
\hline 8912 & - & 0.210 & 0.105 & 0.042 & 0.055 & 0.056 & 0.033 & -0.001 & 0.011 \\
\hline Total & -0.056 & -0.067 & -0.036 & -0.020 & -0.017 & -0.006 & -0.007 & -0.006 & -0.008 \\
\hline
\end{tabular}


Table 8: Wind Speed Distribution

Daytime Data (Greater than $5 \mathrm{Wm}^{-2}$ )

\begin{tabular}{|c|c|c|c|c|c|c|c|c|c|c|}
\hline $\begin{array}{c}\text { Date } \\
(\text { YYWK) }\end{array}$ & $\begin{array}{r}0-1 \\
\mathrm{~m} \mathrm{~s}^{-1} \\
\end{array}$ & $\begin{array}{r}1-2 \\
\mathrm{~m} \mathrm{~s}^{-1} \\
\end{array}$ & $\begin{array}{r}2-3 \\
\mathrm{~m} \mathrm{~s}^{-1} \\
\end{array}$ & $\begin{array}{r}3-4 \\
\mathrm{~m} \mathrm{~s}^{-1} \\
\end{array}$ & $\begin{array}{r}4-5 \\
\mathrm{~m} \mathrm{~s}^{-1} \\
\end{array}$ & $\begin{array}{r}5-6 \\
\mathrm{~m} \mathrm{~s}^{-1} \\
\end{array}$ & $\begin{array}{r}6-7 \\
\mathrm{~m} \mathrm{~s} \mathrm{~s}^{-1} \\
\end{array}$ & $\begin{array}{r}7-8 \\
\mathrm{~m} \mathrm{~s}^{-1} \\
\end{array}$ & $\begin{array}{r}>8 \\
\mathrm{~m} \mathrm{~s}^{-1}\end{array}$ & Total \\
\hline 8825 & 10 & 25 & 17 & 50 & 41 & 26 & 14 & 10 & 5 & 198 \\
\hline 8826 & 5 & 53 & 77 & 63 & 44 & 40 & 50 & 33 & 15 & 380 \\
\hline 8827 & 4 & 18 & 25 & 34 & 43 & 55 & 57 & 44 & 33 & 313 \\
\hline 8828 & 8 & 47 & 99 & 98 & 108 & 117 & 91 & 92 & 54 & 714 \\
\hline 8829 & 2 & 82 & 170 & 153 & 117 & 105 & 70 & 48 & 46 & 793 \\
\hline 8830 & 21 & 92 & 133 & 111 & 114 & 125 & 111 & 64 & 21 & 792 \\
\hline 8831 & 30 & 93 & 111 & 71 & 71 & 107 & 67 & 82 & 158 & 790 \\
\hline 8832 & 2 & 46 & 40 & 57 & 96 & 146 & 136 & 100 & 151 & 774 \\
\hline 8833 & 20 & 89 & 83 & 104 & 74 & 76 & 78 & 35 & 196 & 755 \\
\hline 8834 & 32 & 97 & 148 & 188 & 78 & 68 & 43 & 39 & 43 & 736 \\
\hline 8835 & 1 & 76 & 131 & 141 & 132 & 74 & 76 & 49 & 38 & 718 \\
\hline 8836 & 2 & 46 & 91 & 130 & 93 & 78 & 57 & 56 & 145 & 698 \\
\hline 8837 & 5 & 25 & 68 & 154 & 160 & 106 & 48 & 25 & 98 & 689 \\
\hline 8838 & 7 & 50 & 24 & 28 & 6 & 12 & 11 & 33 & 39 & 210 \\
\hline 8844 & 2 & 10 & 17 & 31 & 47 & 26 & 12 & 12 & 46 & 203 \\
\hline 8845 & 3 & 35 & 59 & 39 & 65 & 73 & 75 & 63 & 144 & 556 \\
\hline 8846 & 1 & 33 & 38 & 70 & 75 & 71 & 69 & 53 & 128 & 538 \\
\hline 8847 & 16 & 23 & 75 & 108 & 103 & 52 & 26 & 30 & 91 & 524 \\
\hline 8848 & 1 & 14 & 24 & 8 & 23 & 48 & 69 & 99 & 219 & 505 \\
\hline 8849 & 0 & 7 & 47 & 72 & 49 & 72 & 54 & 48 & 167 & 516 \\
\hline 8850 & 0 & 0 & 10 & 29 & 61 & 89 & 112 & 83 & 125 & 509 \\
\hline 8851 & 0 & 1 & 20 & 35 & 37 & 56 & 68 & 56 & 227 & 500 \\
\hline 8852 & 0 & 0 & 9 & 41 & 67 & 75 & 60 & 50 & 203 & 505 \\
\hline 8901 & 0 & 0 & 4 & 6 & 26 & 43 & 66 & 89 & 277 & 511 \\
\hline 8902 & 0 & 1 & 3 & 22 & 27 & 46 & 95 & 70 & 248 & 512 \\
\hline 8903 & 0 & 0 & 3 & 27 & 58 & 73 & 75 & 73 & 223 & 532 \\
\hline 8904 & 10 & 80 & 71 & 53 & 71 & 62 & 42 & 35 & 116 & 540 \\
\hline 8905 & 0 & 6 & 26 & 61 & 99 & 137 & 114 & 55 & 50 & 548 \\
\hline 8906 & 14 & 36 & 43 & 71 & 60 & 45 & 38 & 20 & 242 & 569 \\
\hline 8907 & 0 & 0 & 13 & 59 & 84 & 131 & 109 & 72 & 114 & 582 \\
\hline 8908 & 1 & 25 & 29 & 58 & 44 & 50 & 62 & 70 & 246 & 585 \\
\hline 8909 & 1 & 17 & 54 & 101 & 56 & 14 & 41 & 57 & 283 & 624 \\
\hline 8910 & 1 & 2 & 3 & 19 & 23 & 37 & 52 & 60 & 438 & 635 \\
\hline 8911 & 0 & 25 & 96 & 80 & 66 & 50 & 58 & 54 & 223 & 652 \\
\hline 8912 & 0 & 1 & 8 & 31 & 38 & 56 & 62 & 83 & 164 & 443 \\
\hline Total & 199 & 1155 & 1869 & 2403 & 2356 & 2441 & 2268 & 1942 & 5016 & 19649 \\
\hline
\end{tabular}


Table 9: Comparison of Aluminum and Plastic Multi-Plate Shields (VAWR-705-VAWR-121) All Data

\begin{tabular}{|c|c|c|c|c|c|c|c|}
\hline \multirow[b]{2}{*}{$\begin{array}{c}\text { Date } \\
(\mathrm{YYWK})\end{array}$} & \multirow[b]{2}{*}{ Recs } & \multicolumn{3}{|c|}{ Average } & \multicolumn{3}{|c|}{ Standard Deviation } \\
\hline & & $\begin{array}{c}\text { Alum } \\
\text { (C) }\end{array}$ & $\begin{array}{l}\text { Plas } \\
\text { (C) }\end{array}$ & $\begin{array}{l}\mathrm{Al}-\mathrm{Pl} \\
\text { (C) }\end{array}$ & $\begin{array}{l}\text { Alum } \\
\text { (C) }\end{array}$ & $\begin{array}{l}\text { Plas } \\
\text { (C) }\end{array}$ & $\begin{array}{c}\text { Al-Pl } \\
\text { (C) }\end{array}$ \\
\hline 8807 & 828 & 2.660 & 2.654 & 0.007 & 2.463 & 2.397 & 0.197 \\
\hline 8808 & 1345 & 0.473 & 0.510 & -0.037 & 3.598 & 3.588 & 0.107 \\
\hline 8809 & 1345 & 0.992 & 0.992 & -0.001 & 2.843 & 2.866 & 0.183 \\
\hline 8810 & 1345 & 3.456 & 3.490 & -0.034 & 2.324 & 2.311 & 0.081 \\
\hline 8811 & 1345 & 3.059 & 3.067 & -0.008 & 1.843 & 1.852 & 0.087 \\
\hline 8812 & 1345 & 2.317 & 2.346 & -0.029 & 5.345 & 5.335 & 0.081 \\
\hline 8813 & 1345 & 6.462 & 6.471 & -0.009 & 2.408 & 2.378 & 0.110 \\
\hline 8814 & 1345 & 6.717 & 6.710 & 0.007 & 2.554 & 2.506 & 0.100 \\
\hline 8815 & 1345 & 4.994 & 5.007 & -0.013 & 2.607 & 2.591 & 0.053 \\
\hline 8816 & 1345 & 6.714 & 6.734 & -0.020 & 1.866 & 1.868 & 0.058 \\
\hline 8817 & 1345 & 8.657 & 8.653 & 0.004 & 1.495 & 1.490 & 0.095 \\
\hline 8818 & 1345 & 9.405 & 9.407 & -0.002 & 2.763 & 2.760 & 0.070 \\
\hline 8819 & 1345 & 10.627 & 10.609 & 0.018 & 2.581 & 2.529 & 0.124 \\
\hline 8820 & 1345 & 12.444 & 12.430 & 0.014 & 2.194 & 2.094 & 0.178 \\
\hline 8821 & 1345 & 13.209 & 13.264 & -0.055 & 1.801 & 1.819 & 0.104 \\
\hline 8822 & 1345 & 14.165 & 14.197 & -0.032 & 3.521 & 3.526 & 0.163 \\
\hline 8823 & 1345 & 15.189 & 15.289 & -0.100 & 2.942 & 2.977 & 0.137 \\
\hline 8824 & 1345 & 18.401 & 18.432 & -0.031 & 1.983 & 1.991 & 0.161 \\
\hline 8825 & 1345 & 17.231 & 17.236 & -0.004 & 2.213 & 2.189 & 0.129 \\
\hline 8826 & 1345 & 16.687 & 16.691 & -0.004 & 1.854 & 1.830 & 0.189 \\
\hline 8827 & 1345 & 19.179 & 19.280 & -0.101 & 1.772 & 1.837 & 0.150 \\
\hline 8828 & 1345 & 21.114 & 21.135 & -0.021 & 1.680 & 1.644 & 0.143 \\
\hline 8829 & 1345 & 21.469 & 21.482 & -0.013 & 1.687 & 1.670 & 0.105 \\
\hline Total & 30418 & 10.373 & 10.394 & -0.021 & 2.587 & 2.576 & 0.127 \\
\hline
\end{tabular}


Table 10: Comparison of Aluminum and Plastic Multi-Plate Shields (VAWR-705-VAWR-121) Night time Data (less than $5 \mathrm{Wm}^{-2}$ )

\begin{tabular}{|c|c|c|c|c|c|c|c|}
\hline \multirow[b]{2}{*}{$\begin{array}{c}\text { Date } \\
\text { (YYWK) }\end{array}$} & \multirow[b]{2}{*}{ Recs } & \multicolumn{3}{|c|}{ Average } & \multicolumn{3}{|c|}{ Standard Deviation } \\
\hline & & $\begin{array}{l}\text { Alum } \\
\text { (C) }\end{array}$ & $\begin{array}{l}\text { Plas } \\
\text { (C) }\end{array}$ & $\begin{array}{c}\text { Al-Pl } \\
\text { (C) }\end{array}$ & $\begin{array}{l}\text { Alum } \\
\text { (C) }\end{array}$ & $\begin{array}{l}\text { Plas } \\
\text { (C) }\end{array}$ & $\begin{array}{l}\text { Al-Pl } \\
\text { (C) }\end{array}$ \\
\hline 8807 & 445 & 2.148 & 2.151 & -0.003 & 2.434 & 2.374 & 0.218 \\
\hline 8808 & 739 & 0.019 & 0.057 & -0.039 & 4.031 & 4.006 & 0.092 \\
\hline 8809 & 722 & 0.497 & 0.486 & 0.011 & 2.845 & 2.877 & 0.223 \\
\hline 8810 & 694 & 2.822 & 2.843 & -0.021 & 2.265 & 2.241 & 0.050 \\
\hline 8811 & 698 & 2.739 & 2.751 & -0.012 & 1.638 & 1.639 & 0.043 \\
\hline 8812 & 668 & 1.866 & 1.887 & -0.021 & 5.215 & 5.226 & 0.044 \\
\hline 8813 & 648 & 5.717 & 5.747 & -0.030 & 2.368 & 2.328 & 0.075 \\
\hline 8814 & 640 & 6.014 & 6.028 & -0.013 & 1.913 & 1.910 & 0.047 \\
\hline 8815 & 613 & 4.763 & 4.780 & -0.016 & 2.737 & 2.722 & 0.039 \\
\hline 8816 & 600 & 6.204 & 6.218 & -0.014 & 1.453 & 1.459 & 0.034 \\
\hline 8817 & 583 & 7.738 & 7.754 & -0.015 & 1.205 & 1.207 & 0.054 \\
\hline 8818 & 577 & 8.581 & 8.602 & -0.021 & 2.208 & 2.203 & 0.034 \\
\hline 8819 & 547 & 9.306 & 9.344 & -0.038 & 2.475 & 2.456 & 0.071 \\
\hline 8820 & 557 & 11.203 & 11.249 & -0.046 & 1.593 & 1.536 & 0.094 \\
\hline 8821 & 531 & 12.579 & 12.578 & 0.000 & 1.366 & 1.369 & 0.032 \\
\hline 8822 & 522 & 13.260 & 13.333 & -0.073 & 2.647 & 2.674 & 0.136 \\
\hline 8823 & 502 & 13.871 & 13.890 & -0.018 & 2.614 & 2.596 & 0.075 \\
\hline 8824 & 504 & 16.999 & 17.001 & -0.002 & 1.408 & 1.404 & 0.031 \\
\hline 8825 & 504 & 16.526 & 16.549 & -0.023 & 1.817 & 1.807 & 0.041 \\
\hline 8826 & 512 & 15.846 & 15.836 & 0.009 & 1.546 & 1.521 & 0.123 \\
\hline 8827 & 507 & 18.079 & 18.082 & -0.003 & 1.266 & 1.264 & 0.053 \\
\hline 8828 & 521 & 19.852 & 19.855 & -0.003 & 0.726 & 0.716 & 0.030 \\
\hline 8829 & 557 & 20.852 & 20.866 & -0.014 & 1.370 & 1.341 & 0.052 \\
\hline Total & 13391 & 8.822 & 8.840 & -0.018 & 2.418 & 2.410 & 0.090 \\
\hline
\end{tabular}


Table 11: Comparison of Aluminum and Plastic Multi-Plate Shields (VAWR-705-VAWR-121) Daytime Data (Greater than 5 Wm$^{-2}$ )

\begin{tabular}{|c|c|c|c|c|c|c|c|}
\hline \multirow[b]{2}{*}{$\begin{array}{c}\text { Date } \\
\text { (YYWK) }\end{array}$} & \multirow[b]{2}{*}{ Recs } & \multicolumn{3}{|c|}{ Average } & \multicolumn{3}{|c|}{ Standard Deviation } \\
\hline & & $\begin{array}{c}\text { Alum } \\
\text { (C) }\end{array}$ & $\begin{array}{l}\text { Plas } \\
\text { (C) }\end{array}$ & $\begin{array}{c}\text { Al-Pl } \\
\text { (C) }\end{array}$ & $\begin{array}{c}\text { Alum } \\
\text { (C) }\end{array}$ & $\begin{array}{l}\text { Plas } \\
\text { (C) }\end{array}$ & $\begin{array}{l}\mathrm{Al}-\mathrm{Pl} \\
\text { (C) }\end{array}$ \\
\hline 8807 & 383 & 3.255 & 3.237 & 0.018 & 2.364 & 2.291 & 0.169 \\
\hline 8808 & 606 & 1.027 & 1.062 & -0.035 & 2.894 & 2.910 & 0.123 \\
\hline 8809 & 623 & 1.565 & 1.579 & -0.014 & 2.733 & 2.741 & 0.117 \\
\hline 8810 & 651 & 4.132 & 4.180 & -0.048 & 2.192 & 2.182 & 0.102 \\
\hline 8811 & 647 & 3.403 & 3.407 & -0.004 & 1.985 & 2.004 & 0.118 \\
\hline 8812 & 677 & 2.762 & 2.798 & -0.037 & 5.438 & 5.407 & 0.105 \\
\hline 8813 & 697 & 7.154 & 7.144 & 0.010 & 2.235 & 2.222 & 0.132 \\
\hline 8814 & 705 & 7.356 & 7.330 & 0.026 & 2.877 & 2.806 & 0.129 \\
\hline 8815 & 732 & 5.188 & 5.197 & -0.010 & 2.479 & 2.461 & 0.062 \\
\hline 8816 & 745 & 7.124 & 7.150 & -0.026 & 2.053 & 2.050 & 0.072 \\
\hline 8817 & 762 & 9.360 & 9.341 & 0.019 & 1.302 & 1.309 & 0.114 \\
\hline 8818 & 768 & 10.024 & 10.012 & 0.013 & 2.971 & 2.974 & 0.085 \\
\hline 8819 & 798 & 11.532 & 11.476 & 0.056 & 2.240 & 2.192 & 0.137 \\
\hline 8820 & 788 & 13.320 & 13.264 & 0.056 & 2.138 & 2.034 & 0.208 \\
\hline 8821 & 814 & 13.620 & 13.710 & -0.090 & 1.929 & 1.935 & 0.119 \\
\hline 8822 & 823 & 14.739 & 14.745 & -0.006 & 3.871 & 3.876 & 0.174 \\
\hline 8823 & 843 & 15.974 & 16.122 & -0.148 & 2.845 & 2.876 & 0.143 \\
\hline 8824 & 841 & 19.242 & 19.289 & -0.048 & 1.793 & 1.789 & 0.201 \\
\hline 8825 & 841 & 17.654 & 17.647 & 0.007 & 2.320 & 2.293 & 0.158 \\
\hline 8826 & 833 & 17.204 & 17.217 & -0.012 & 1.839 & 1.806 & 0.220 \\
\hline 8827 & 838 & 19.844 & 20.004 & -0.160 & 1.702 & 1.749 & 0.158 \\
\hline 8828 & 824 & 21.912 & 21.945 & -0.033 & 1.622 & 1.548 & 0.180 \\
\hline 8829 & 788 & 21.905 & 21.917 & -0.012 & 1.753 & 1.742 & 0.130 \\
\hline Total & 17027 & 11.593 & 11.616 & -0.023 & 2.534 & 2.519 & 0.145 \\
\hline
\end{tabular}


Table 12: Mean Differences of Aluminum minus Plastic

Gill Multi-Plate Shields by Wind Speed

(VAWR-705-VAWR-121) All Data

\begin{tabular}{crrrrrrrrr}
$\begin{array}{c}\text { Date } \\
\text { (YYWK) }\end{array}$ & $\begin{array}{r}0-1 \\
\mathrm{~m} \mathrm{~s}^{-1}\end{array}$ & $\begin{array}{r}1-2 \\
\mathrm{~m} \mathrm{~s}^{-1}\end{array}$ & $\begin{array}{r}\mathrm{m} \mathrm{s}^{-1} \\
\mathrm{~m} \mathrm{~s}^{-1}\end{array}$ & $\mathrm{~m} \mathrm{~s}^{-1}$ & $\begin{array}{r}5-6 \\
\mathrm{~m} \mathrm{~s}^{-1}\end{array}$ & $\mathrm{~m} \mathrm{~s}^{-1}$ & $\mathrm{~m} \mathrm{~s}^{-1}$ & $\mathrm{~m} \mathrm{~s}^{-1}$ \\
\hline 8807 & -0.223 & -0.082 & -0.006 & 0.084 & 0.072 & 0.090 & -0.035 & -0.013 & 0.093 \\
8808 & -0.155 & -0.057 & 0.020 & -0.015 & -0.028 & -0.025 & -0.015 & -0.018 & -0.058 \\
8809 & -0.170 & -0.048 & -0.048 & -0.014 & 0.083 & 0.046 & -0.006 & -0.010 & -0.015 \\
8810 & -0.158 & -0.015 & -0.023 & -0.022 & -0.027 & -0.011 & -0.038 & -0.064 & -0.049 \\
8811 & -0.022 & -0.026 & -0.009 & -0.007 & -0.000 & -0.007 & -0.001 & 0.019 & -0.008 \\
8812 & 0.022 & -0.054 & 0.000 & -0.034 & -0.050 & -0.045 & -0.044 & -0.039 & -0.011 \\
8813 & -0.043 & 0.011 & -0.013 & 0.002 & -0.002 & -0.047 & -0.041 & -0.019 & 0.049 \\
8814 & 0.056 & 0.040 & 0.050 & 0.021 & -0.005 & -0.010 & -0.003 & -0.007 & -0.007 \\
8815 & - & -0.033 & -0.036 & -0.005 & -0.000 & -0.009 & -0.015 & -0.019 & -0.023 \\
8816 & -0.017 & -0.013 & 0.005 & -0.011 & -0.023 & -0.019 & -0.020 & -0.022 & -0.030 \\
8817 & -0.080 & 0.021 & -0.005 & 0.000 & 0.019 & 0.020 & 0.001 & -0.006 & -0.001 \\
8818 & 0.011 & 0.040 & 0.001 & 0.002 & -0.011 & -0.011 & -0.012 & 0.002 & -0.018 \\
8819 & -0.126 & -0.053 & 0.051 & 0.040 & 0.040 & 0.025 & 0.008 & -0.038 & -0.018 \\
8820 & -0.109 & -0.006 & 0.084 & 0.068 & 0.022 & 0.004 & -0.050 & -0.142 & -0.111 \\
8821 & -0.048 & -0.030 & -0.040 & -0.027 & -0.043 & -0.038 & -0.048 & -0.080 & -0.119 \\
8822 & -0.234 & -0.078 & -0.032 & 0.023 & 0.029 & 0.000 & -0.013 & 0.013 & 0.000 \\
8823 & -0.166 & -0.127 & -0.088 & -0.088 & -0.087 & -0.116 & -0.081 & -0.113 & -0.086 \\
8824 & -0.050 & -0.045 & 0.023 & -0.002 & -0.061 & -0.063 & -0.041 & -0.039 & -0.134 \\
8825 & 0.021 & -0.007 & 0.046 & 0.039 & 0.022 & -0.026 & -0.068 & -0.054 & -0.039 \\
8826 & -0.147 & -0.068 & 0.022 & 0.010 & 0.008 & -0.038 & 0.008 & -0.005 & 0.060 \\
8827 & -0.097 & -0.069 & -0.059 & -0.046 & -0.082 & -0.086 & -0.178 & -0.216 & -0.235 \\
8828 & -0.039 & -0.030 & 0.013 & 0.027 & -0.008 & -0.000 & -0.031 & -0.124 & -0.171 \\
8829 & -0.034 & -0.067 & -0.024 & 0.013 & 0.012 & -0.016 & -0.037 & -0.013 & 0.020 \\
& & & & & & & & & \\
Total & -0.101 & -0.037 & -0.004 & 0.004 & -0.002 & -0.017 & -0.034 & -0.042 & -0.034 \\
\hline & & & & & & & & &
\end{tabular}


Table 13: Wind Speed Distribution

\section{All Data}

\begin{tabular}{crrrrrrrrrr}
$\begin{array}{c}\text { Date } \\
\text { (YYWK) }\end{array}$ & $\begin{array}{r}0-1 \\
\mathrm{~m} \mathrm{~s}^{-1}\end{array}$ & $\begin{array}{r}1-2 \\
\mathrm{~m} \mathrm{~s}^{-1}\end{array}$ & $\begin{array}{r}2-3 \\
\mathrm{~m} \mathrm{~s}^{-1}\end{array}$ & $\begin{array}{r}3-4 \\
\mathrm{~m} \mathrm{~s}^{-1}\end{array}$ & $\begin{array}{r}4-5 \\
\mathrm{~m} \mathrm{~s}^{-1}\end{array}$ & $\begin{array}{r}5-6 \\
\mathrm{~m} \mathrm{~s}^{-1}\end{array}$ & $\begin{array}{r}6-7 \\
\mathrm{~m} \mathrm{~s}^{-1}\end{array}$ & $\begin{array}{r}7-8 \\
\mathrm{~m} \mathrm{~s}^{-1}\end{array}$ & $\begin{array}{r}>8 \\
\mathrm{~m} \mathrm{~s}^{-1}\end{array}$ & Total \\
\hline 8807 & 80 & 117 & 149 & 125 & 93 & 83 & 36 & 30 & 115 & 828 \\
8808 & 50 & 191 & 162 & 165 & 103 & 116 & 77 & 78 & 403 & 1345 \\
8809 & 74 & 68 & 171 & 184 & 232 & 222 & 184 & 101 & 109 & 1345 \\
8810 & 26 & 50 & 155 & 211 & 215 & 191 & 151 & 176 & 170 & 1345 \\
8811 & 51 & 169 & 257 & 235 & 236 & 166 & 55 & 49 & 127 & 1345 \\
8812 & 48 & 84 & 134 & 102 & 102 & 178 & 196 & 167 & 334 & 1345 \\
8813 & 161 & 227 & 213 & 211 & 112 & 132 & 101 & 56 & 132 & 1345 \\
8814 & 54 & 104 & 119 & 130 & 124 & 163 & 186 & 161 & 304 & 1345 \\
8815 & 0 & 20 & 132 & 261 & 265 & 206 & 180 & 146 & 135 & 1345 \\
8816 & 11 & 46 & 108 & 141 & 153 & 166 & 176 & 137 & 407 & 1345 \\
8817 & 27 & 136 & 201 & 235 & 196 & 154 & 126 & 123 & 147 & 1345 \\
8818 & 31 & 141 & 147 & 211 & 237 & 205 & 125 & 100 & 148 & 1345 \\
8819 & 45 & 171 & 166 & 278 & 344 & 220 & 81 & 23 & 17 & 1345 \\
8820 & 163 & 280 & 237 & 261 & 211 & 132 & 37 & 17 & 7 & 1345 \\
8821 & 48 & 127 & 173 & 160 & 192 & 171 & 156 & 103 & 215 & 1345 \\
8822 & 134 & 191 & 273 & 315 & 194 & 114 & 68 & 42 & 14 & 1345 \\
8823 & 57 & 191 & 216 & 223 & 202 & 113 & 100 & 60 & 183 & 1345 \\
8824 & 76 & 267 & 307 & 205 & 139 & 135 & 93 & 53 & 70 & 1345 \\
8825 & 22 & 131 & 177 & 221 & 219 & 163 & 176 & 109 & 127 & 1345 \\
8826 & 22 & 148 & 207 & 263 & 233 & 167 & 125 & 96 & 84 & 1345 \\
8827 & 46 & 165 & 231 & 168 & 186 & 241 & 148 & 94 & 66 & 1345 \\
8828 & 23 & 120 & 199 & 216 & 223 & 228 & 150 & 117 & 69 & 1345 \\
8829 & 12 & 161 & 269 & 237 & 228 & 160 & 100 & 98 & 80 & 1345 \\
& & & & & & & & & & \\
Total & 1261 & 3305 & 4403 & 4758 & 4439 & 3826 & 2827 & 2136 & 3463 & 30418 \\
\hline & & & & & & & & & &
\end{tabular}


Table 14: Mean Differences of Aluminum minus Plastic

Gill Multi-Plate Shields by Wind Speed

(VAWR-705-VAWR-121) Daytime Data (Greater than $5 \mathrm{Wm}^{-2}$ )

\begin{tabular}{crrrrrrrrr}
$\begin{array}{c}\text { Date } \\
\text { (YYWK) }\end{array}$ & $\begin{array}{r}0-1 \\
\mathrm{~m} \mathrm{~s}^{-1}\end{array}$ & $\begin{array}{r}1-2 \\
\mathrm{~m} \mathrm{~s}^{-1}\end{array}$ & $\begin{array}{r}2-3 \\
\mathrm{~m} \mathrm{~s}^{-1}\end{array}$ & $\begin{array}{r}3-4 \\
\mathrm{~m} \mathrm{~s}^{-1}\end{array}$ & $\begin{array}{r}4-5 \\
\mathrm{~m} \mathrm{~s}^{-1}\end{array}$ & $\begin{array}{r}5-6 \\
\mathrm{~m} \mathrm{~s}^{-1}\end{array}$ & $\begin{array}{r}6-7 \\
\mathrm{~m} \mathrm{~s}^{-1}\end{array}$ & $\begin{array}{r}7-8 \\
\mathrm{~m} \mathrm{~s}^{-1}\end{array}$ & $\begin{array}{r}>8 \\
\mathrm{~m} \mathrm{~s}^{-1}\end{array}$ \\
\hline 8807 & -0.053 & -0.041 & -0.003 & 0.016 & 0.125 & -0.014 & -0.137 & -0.045 & 0.109 \\
8808 & -0.022 & 0.012 & 0.056 & 0.004 & -0.044 & -0.038 & -0.063 & -0.070 & -0.093 \\
8809 & 0.063 & 0.082 & -0.017 & -0.025 & -0.020 & 0.019 & -0.016 & -0.030 & -0.084 \\
8810 & -0.152 & 0.117 & 0.006 & -0.016 & -0.049 & -0.029 & -0.085 & -0.091 & -0.090 \\
8811 & 0.021 & -0.017 & -0.008 & -0.009 & 0.008 & -0.015 & 0.014 & 0.031 & -0.010 \\
8812 & 0.180 & -0.034 & 0.023 & -0.019 & -0.100 & -0.079 & -0.069 & -0.066 & -0.021 \\
8813 & 0.053 & 0.087 & 0.027 & 0.017 & 0.004 & -0.087 & -0.057 & -0.021 & 0.012 \\
8814 & 0.224 & 0.161 & 0.125 & 0.055 & -0.002 & -0.010 & -0.005 & -0.008 & -0.008 \\
8815 & - & - & 0.038 & 0.013 & 0.012 & -0.012 & -0.018 & -0.026 & -0.033 \\
8816 & 0.250 & 0.022 & 0.052 & 0.022 & -0.026 & -0.033 & -0.033 & -0.036 & -0.055 \\
8817 & -0.080 & 0.072 & 0.034 & 0.015 & 0.047 & 0.027 & 0.005 & -0.010 & -0.004 \\
8818 & 0.050 & 0.102 & 0.058 & 0.028 & -0.003 & -0.006 & -0.013 & 0.005 & -0.020 \\
8819 & -0.152 & -0.029 & 0.147 & 0.088 & 0.062 & 0.034 & 0.014 & -0.047 & -0.029 \\
8820 & -0.088 & 0.034 & 0.138 & 0.103 & 0.046 & 0.021 & -0.065 & -0.176 & -0.111 \\
8821 & -0.080 & -0.044 & -0.065 & -0.058 & -0.080 & -0.081 & -0.089 & -0.110 & -0.129 \\
8822 & -0.195 & -0.041 & -0.033 & 0.041 & 0.062 & 0.002 & -0.006 & 0.017 & 0.003 \\
8823 & -0.273 & -0.210 & -0.118 & -0.127 & -0.144 & -0.148 & -0.122 & -0.164 & -0.159 \\
8824 & -0.080 & -0.056 & 0.040 & -0.002 & -0.099 & -0.095 & -0.073 & -0.071 & -0.181 \\
8825 & 0.053 & 0.024 & 0.117 & 0.085 & 0.049 & -0.022 & -0.082 & -0.079 & -0.071 \\
8826 & -0.171 & -0.117 & 0.008 & 0.009 & 0.012 & -0.047 & 0.018 & -0.003 & 0.067 \\
8827 & -0.135 & -0.126 & -0.113 & -0.097 & -0.154 & -0.153 & -0.203 & -0.216 & -0.235 \\
8828 & -0.072 & -0.033 & 0.039 & 0.051 & -0.020 & -0.008 & -0.053 & -0.138 & -0.171 \\
8829 & 0.018 & -0.102 & -0.022 & 0.031 & 0.023 & -0.028 & -0.067 & -0.025 & 0.031 \\
& & & & & & & & & \\
Total & -0.037 & -0.018 & 0.014 & 0.016 & -0.006 & -0.035 & -0.054 & -0.062 & -0.061 \\
\hline & & & & & & & & &
\end{tabular}


Table 15: Wind Speed Distribution

Daytime Data (Greater than $5 \mathrm{Wm}^{-2}$ )

\begin{tabular}{crrrrrrrrrr}
$\begin{array}{c}\text { Date } \\
\text { (YYWK) }\end{array}$ & $\begin{array}{r}0-1 \\
\mathrm{~m} \mathrm{~s}^{-1}\end{array}$ & $\begin{array}{rrrrr}1-2 \\
\mathrm{~m} \mathrm{~s}^{-1}\end{array}$ & $\begin{array}{r}2-3 \\
\mathrm{~m} \mathrm{~s}^{-1}\end{array}$ & $\begin{array}{r}3-4 \\
\mathrm{~m} \mathrm{~s}^{-1}\end{array}$ & $\begin{array}{r}4-5 \\
\mathrm{~m} \mathrm{~s}^{-1}\end{array}$ & $\begin{array}{r}5-6 \\
\mathrm{~m} \mathrm{~s}^{-1}\end{array}$ & $\begin{array}{r}6-7 \\
\mathrm{~m} \mathrm{~s}^{-1}\end{array}$ & $\begin{array}{r}7-8 \\
\mathrm{~m} \mathrm{~s}^{-1}\end{array}$ & $\mathrm{~m} \mathrm{~s}^{-1}$ & Total \\
\hline 8807 & 17 & 42 & 81 & 70 & 41 & 25 & 22 & 17 & 68 & 383 \\
8808 & 12 & 80 & 102 & 69 & 35 & 24 & 22 & 23 & 239 & 606 \\
8809 & 13 & 21 & 53 & 90 & 138 & 105 & 101 & 54 & 48 & 623 \\
8810 & 7 & 20 & 77 & 115 & 87 & 63 & 64 & 125 & 93 & 651 \\
8811 & 30 & 83 & 147 & 114 & 96 & 51 & 23 & 31 & 72 & 647 \\
8812 & 16 & 37 & 77 & 29 & 25 & 87 & 117 & 93 & 196 & 677 \\
8813 & 60 & 112 & 97 & 124 & 61 & 74 & 74 & 50 & 45 & 697 \\
8814 & 31 & 36 & 51 & 51 & 45 & 72 & 111 & 117 & 191 & 705 \\
8815 & 0 & 0 & 8 & 103 & 132 & 131 & 143 & 110 & 105 & 732 \\
8816 & 1 & 23 & 59 & 66 & 98 & 87 & 95 & 79 & 237 & 745 \\
8817 & 27 & 87 & 74 & 78 & 96 & 111 & 106 & 87 & 96 & 762 \\
8818 & 21 & 78 & 49 & 79 & 149 & 98 & 100 & 70 & 124 & 768 \\
8819 & 5 & 59 & 83 & 164 & 250 & 159 & 52 & 19 & 7 & 798 \\
8820 & 39 & 131 & 151 & 187 & 141 & 90 & 28 & 14 & 7 & 788 \\
8821 & 10 & 50 & 97 & 74 & 107 & 97 & 101 & 78 & 200 & 814 \\
8822 & 47 & 116 & 200 & 208 & 110 & 60 & 38 & 32 & 12 & 823 \\
8823 & 25 & 83 & 147 & 152 & 125 & 90 & 71 & 42 & 108 & 843 \\
8824 & 42 & 178 & 185 & 112 & 87 & 94 & 60 & 29 & 54 & 841 \\
8825 & 14 & 57 & 91 & 128 & 146 & 121 & 137 & 78 & 69 & 841 \\
8826 & 17 & 91 & 129 & 142 & 123 & 112 & 95 & 73 & 51 & 833 \\
8827 & 9 & 77 & 122 & 92 & 103 & 144 & 131 & 94 & 66 & 838 \\
8828 & 10 & 52 & 111 & 121 & 125 & 133 & 96 & 107 & 69 & 824 \\
8829 & 4 & 63 & 169 & 152 & 136 & 104 & 62 & 59 & 39 & 788 \\
& & & & & & & & & & \\
Total & 457 & 1576 & 2360 & 2520 & 2456 & 2132 & 1849 & 1481 & 2196 & 17027 \\
\hline & & & & & & & & & &
\end{tabular}


Table 16: Comparison of Aluminum and Plastic Multi-Plate (with solar fan) Shields (VAWR-705-VAWR-121) All Data

\begin{tabular}{cccccccc} 
& & \multicolumn{3}{c}{ Average } & \multicolumn{3}{c}{ Standard Deviation } \\
$\begin{array}{c}\text { Date } \\
\text { (YYWK) }\end{array}$ & Recs & $\begin{array}{c}\text { Alum } \\
\text { Ylas }\end{array}$ & $\begin{array}{c}\text { Pl-Pl } \\
(\mathrm{C})\end{array}$ & $\begin{array}{c}\text { Alum } \\
(\mathrm{C})\end{array}$ & $\begin{array}{c}\text { Plas } \\
(\mathrm{C})\end{array}$ & $\begin{array}{c}\text { Al-Pl } \\
(\mathrm{C})\end{array}$ & $(\mathrm{C})$ \\
\hline & & & & & & & \\
8830 & 1034 & 22.015 & 22.105 & -0.089 & 1.182 & 1.248 & 0.208 \\
8831 & 1345 & 23.291 & 23.375 & -0.084 & 1.279 & 1.344 & 0.175 \\
8832 & 1345 & 23.744 & 23.846 & -0.103 & 0.993 & 1.074 & 0.142 \\
8833 & 1345 & 21.545 & 21.597 & -0.052 & 1.995 & 2.015 & 0.154 \\
8834 & 1345 & 18.720 & 18.764 & -0.043 & 2.404 & 2.384 & 0.203 \\
8835 & 1345 & 20.358 & 20.408 & -0.051 & 2.157 & 2.183 & 0.156 \\
8836 & 1345 & 18.549 & 18.612 & -0.064 & 2.209 & 2.196 & 0.158 \\
8837 & 1345 & 16.769 & 16.837 & -0.068 & 2.287 & 2.310 & 0.152 \\
8838 & 1142 & 18.535 & 18.607 & -0.072 & 1.426 & 1.484 & 0.175 \\
8839 & 1345 & 15.640 & 15.693 & -0.053 & 2.320 & 2.346 & 0.162 \\
8840 & 1345 & 12.134 & 12.192 & -0.059 & 4.059 & 4.057 & 0.107 \\
8841 & 1345 & 10.486 & 10.552 & -0.066 & 3.521 & 3.532 & 0.109 \\
8842 & 1345 & 11.695 & 11.719 & -0.024 & 3.358 & 3.327 & 0.105 \\
8843 & 876 & 10.667 & 10.701 & -0.034 & 2.187 & 2.198 & 0.096 \\
& & & & & & & \\
Total & 17847 & 17.525 & 17.586 & -0.062 & 2.433 & 2.445 & 0.154 \\
\hline
\end{tabular}


Table 17: Comparison of Aluminum and Plastic Multi-Plate (with solar fan) Shields (VAWR-705-VAWR-121) Night time Data (less than $5 \mathrm{Wm}^{-2}$ )

\begin{tabular}{|c|c|c|c|c|c|c|c|}
\hline \multirow[b]{2}{*}{$\begin{array}{c}\text { Date } \\
\text { (YYWK) }\end{array}$} & \multirow[b]{2}{*}{ Recs } & \multicolumn{3}{|c|}{ Average } & \multicolumn{3}{|c|}{ Standard Deviation } \\
\hline & & $\begin{array}{c}\text { Alum } \\
\text { (C) }\end{array}$ & $\begin{array}{l}\text { Plas } \\
\text { (C) }\end{array}$ & $\begin{array}{c}\text { Al-Pl } \\
\text { (C) }\end{array}$ & $\begin{array}{l}\text { Alum } \\
\text { (C) }\end{array}$ & $\begin{array}{l}\text { Plas } \\
\text { (C) }\end{array}$ & $\begin{array}{c}\text { Al-Pl } \\
\text { (C) }\end{array}$ \\
\hline 8830 & 392 & 21.263 & 21.250 & 0.013 & 0.572 & 0.584 & 0.029 \\
\hline 8831 & 549 & 22.549 & 22.542 & 0.007 & 0.898 & 0.881 & 0.060 \\
\hline 8832 & 569 & 23.175 & 23.163 & 0.012 & 0.658 & 0.658 & 0.026 \\
\hline 8833 & 583 & 21.154 & 21.147 & 0.008 & 2.253 & 2.238 & 0.055 \\
\hline 8834 & 596 & 17.812 & 17.829 & -0.017 & 1.921 & 1.876 & 0.085 \\
\hline 8835 & 621 & 19.695 & 19.692 & 0.003 & 2.106 & 2.090 & 0.066 \\
\hline 8836 & 641 & 18.058 & 18.047 & 0.010 & 1.942 & 1.939 & 0.062 \\
\hline 8837 & 649 & 16.580 & 16.564 & 0.017 & 2.400 & 2.394 & 0.030 \\
\hline 8838 & 575 & 18.149 & 18.148 & 0.000 & 1.242 & 1.233 & 0.050 \\
\hline 8839 & 684 & 14.744 & 14.790 & -0.047 & 2.149 & 2.131 & 0.094 \\
\hline 8840 & 723 & 11.833 & 11.875 & -0.042 & 3.999 & 3.972 & 0.063 \\
\hline 8841 & 718 & 10.117 & 10.102 & 0.014 & 3.691 & 3.697 & 0.026 \\
\hline 8842 & 749 & 11.396 & 11.429 & -0.033 & 3.105 & 3.099 & 0.073 \\
\hline 8843 & 519 & 10.312 & 10.298 & 0.014 & 1.924 & 1.931 & 0.028 \\
\hline Total & 8568 & 16.532 & 16.536 & -0.005 & 2.400 & 2.390 & 0.059 \\
\hline
\end{tabular}


Table 18: Comparison of Aluminum and Plastic Multi-Plate (with solar fan) Shields (VAWR-705-VAWR-121) Daytime Data (Greater than $5 \mathrm{Wm}^{-2}$ )

\begin{tabular}{|c|c|c|c|c|c|c|c|}
\hline \multirow[b]{2}{*}{$\begin{array}{c}\text { Date } \\
(Y Y W K)\end{array}$} & \multirow[b]{2}{*}{ Recs } & \multicolumn{3}{|c|}{ Average } & \multicolumn{3}{|c|}{ Standard Deviation } \\
\hline & & $\begin{array}{c}\text { Alum } \\
\text { (C) }\end{array}$ & $\begin{array}{l}\text { Plas } \\
\text { (C) }\end{array}$ & $\begin{array}{c}\text { Al-Pl } \\
(\mathrm{C})\end{array}$ & $\begin{array}{c}\text { Alum } \\
\text { (C) }\end{array}$ & $\begin{array}{l}\text { Plas } \\
\text { (C) }\end{array}$ & $\begin{array}{c}\text { Al-Pl } \\
\text { (C) }\end{array}$ \\
\hline 8830 & 642 & 22.474 & 22.627 & -0.152 & 1.223 & 1.259 & 0.243 \\
\hline 8831 & 796 & 23.802 & 23.949 & -0.146 & 1.252 & 1.307 & 0.199 \\
\hline 8832 & 776 & 24.160 & 24.347 & -0.187 & 0.991 & 1.043 & 0.133 \\
\hline 8833 & 762 & 21.843 & 21.941 & -0.098 & 1.715 & 1.752 & 0.187 \\
\hline 8834 & 749 & 19.443 & 19.508 & -0.064 & 2.504 & 2.482 & 0.260 \\
\hline 8835 & 724 & 20.926 & 21.023 & -0.096 & 2.037 & 2.073 & 0.192 \\
\hline 8836 & 704 & 18.996 & 19.127 & -0.131 & 2.339 & 2.290 & 0.187 \\
\hline 8837 & 696 & 16.945 & 17.091 & -0.146 & 2.163 & 2.200 & 0.176 \\
\hline 8838 & 567 & 18.926 & 19.073 & -0.146 & 1.493 & 1.570 & 0.220 \\
\hline 8839 & 661 & 16.568 & 16.627 & -0.059 & 2.118 & 2.188 & 0.209 \\
\hline 8840 & 622 & 12.483 & 12.561 & -0.078 & 4.103 & 4.126 & 0.139 \\
\hline 8841 & 627 & 10.910 & 11.067 & -0.157 & 3.267 & 3.260 & 0.094 \\
\hline 8842 & 596 & 12.070 & 12.083 & -0.013 & 3.620 & 3.561 & 0.133 \\
\hline 8843 & 357 & 11.182 & 11.285 & -0.103 & 2.432 & 2.422 & 0.115 \\
\hline Total & 9279 & 18.442 & 18.556 & -0.114 & 2.350 & 2.361 & 0.168 \\
\hline
\end{tabular}


Table 19: Mean Differences of Aluminum minus Plastic (with solar fan) Gill Multi-Plate Shields by Wind Speed

(VAWR-705-VAWR-121) All Data

\begin{tabular}{ccrrrrrrrr}
$\begin{array}{c}\text { Date } \\
(Y Y W K)\end{array}$ & $\begin{array}{r}0-1 \\
\mathrm{~m} \mathrm{~s}^{-1}\end{array}$ & $\begin{array}{r}1-2 \\
\mathrm{~m} \mathrm{~s}^{-1}\end{array}$ & $\begin{array}{r}2-3 \\
\mathrm{~m} \mathrm{~s}^{-1}\end{array}$ & $\begin{array}{r}3-4 \\
\mathrm{~m} \mathrm{~s}^{-1}\end{array}$ & $\begin{array}{r}4-5 \\
\mathrm{~m} \mathrm{~s}^{-1}\end{array}$ & $\begin{array}{r}5-6 \\
\mathrm{~m} \mathrm{~s}^{-1}\end{array}$ & $\begin{array}{r}6-7 \\
\mathrm{~m} \mathrm{~s}^{-1}\end{array}$ & $\begin{array}{r}7-8 \\
\mathrm{~m} \mathrm{~s}^{-1}\end{array}$ & $\begin{array}{r}>8 \\
\mathrm{~m} \mathrm{~s}^{-1}\end{array}$ \\
\hline 8830 & -0.232 & -0.104 & -0.071 & -0.042 & -0.077 & -0.071 & -0.154 & -0.271 & -0.120 \\
8831 & -0.090 & -0.061 & -0.011 & -0.023 & -0.033 & -0.069 & -0.126 & -0.159 & -0.234 \\
8832 & -0.157 & -0.155 & -0.044 & -0.044 & -0.075 & -0.114 & -0.088 & -0.105 & -0.212 \\
8833 & -0.149 & -0.141 & -0.024 & 0.014 & -0.043 & -0.053 & -0.041 & -0.053 & -0.066 \\
8834 & -0.096 & -0.092 & -0.002 & -0.010 & -0.060 & -0.086 & -0.067 & -0.070 & -0.013 \\
8835 & -0.106 & -0.101 & -0.034 & -0.013 & -0.056 & -0.051 & -0.065 & -0.096 & -0.019 \\
8836 & -0.054 & -0.078 & -0.067 & -0.044 & -0.050 & -0.097 & -0.052 & -0.037 & -0.084 \\
8837 & -0.144 & -0.307 & -0.167 & -0.062 & -0.058 & -0.052 & -0.070 & -0.030 & -0.033 \\
8838 & -0.153 & -0.189 & -0.059 & -0.059 & -0.100 & -0.042 & -0.019 & -0.039 & -0.059 \\
8839 & -0.208 & -0.091 & -0.044 & 0.006 & -0.055 & -0.048 & -0.023 & -0.045 & -0.115 \\
8840 & -0.289 & -0.131 & -0.076 & -0.055 & -0.054 & -0.030 & -0.062 & -0.071 & -0.078 \\
8841 & - & -0.072 & -0.128 & -0.075 & -0.059 & -0.095 & -0.088 & -0.014 & -0.059 \\
8842 & -0.108 & -0.089 & -0.060 & -0.021 & 0.005 & -0.006 & -0.010 & -0.021 & -0.005 \\
8843 & - & 0.047 & -0.020 & -0.031 & -0.024 & -0.010 & -0.011 & -0.038 & -0.051 \\
& & & & & & & & & \\
Total & -0.135 & -0.111 & -0.046 & -0.030 & -0.050 & -0.062 & -0.065 & -0.071 & -0.079 \\
\hline
\end{tabular}


Table 20: Wind Speed Distribution

All Data

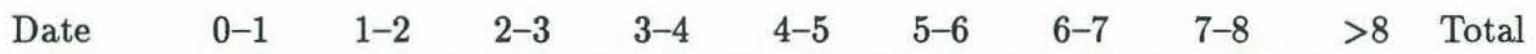
(YYWK) $\mathrm{m} \mathrm{s}^{-1} \mathrm{~m} \mathrm{~s}^{-1} \mathrm{~m} \mathrm{~s}^{-1} \quad \mathrm{~m} \mathrm{~s}^{-1} \quad \mathrm{~m} \mathrm{~s}^{-1} \quad \mathrm{~m} \mathrm{~s}^{-1} \quad \mathrm{~m} \mathrm{~s}^{-1} \mathrm{~m} \mathrm{~s}^{-1} \mathrm{~m} \mathrm{~s}^{-1}$

\begin{tabular}{lrrrrrrrrrr}
8830 & 13 & 120 & 232 & 213 & 161 & 143 & 92 & 50 & 10 & 1034 \\
8831 & 58 & 172 & 181 & 152 & 192 & 191 & 125 & 100 & 174 & 1345 \\
8832 & 2 & 62 & 91 & 180 & 217 & 253 & 214 & 158 & 168 & 1345 \\
8833 & 38 & 140 & 172 & 193 & 153 & 140 & 138 & 65 & 306 & 1345 \\
8834 & 49 & 192 & 333 & 269 & 192 & 134 & 85 & 44 & 47 & 1345 \\
8835 & 18 & 174 & 274 & 236 & 202 & 127 & 111 & 94 & 109 & 1345 \\
8836 & 15 & 78 & 214 & 251 & 197 & 143 & 106 & 95 & 246 & 1345 \\
8837 & 3 & 35 & 80 & 241 & 356 & 299 & 131 & 47 & 153 & 1345 \\
8838 & 47 & 102 & 138 & 195 & 168 & 131 & 138 & 76 & 147 & 1142 \\
8839 & 45 & 97 & 283 & 276 & 127 & 96 & 113 & 112 & 196 & 1345 \\
8840 & 8 & 25 & 118 & 302 & 267 & 237 & 212 & 84 & 92 & 1345 \\
8841 & 0 & 10 & 18 & 79 & 206 & 216 & 221 & 169 & 426 & 1345 \\
8842 & 32 & 92 & 181 & 350 & 225 & 136 & 82 & 55 & 192 & 1345 \\
8843 & 0 & 2 & 45 & 90 & 76 & 117 & 96 & 69 & 381 & 876 \\
& & & & & & & & & & \\
Total & 328 & 1301 & 2360 & 3027 & 2739 & 2363 & 1864 & 1218 & 2647 & 17847 \\
\hline
\end{tabular}


Table 21: Mean Differences of Aluminum minus Plastic (with solar fan) Gill Multi-Plate Shields by Wind Speed (VAWR-705-VAWR-121) Daytime Data (Greater than $5 \mathrm{Wm}^{-2}$ )

\begin{tabular}{crrrrrrrrr}
$\begin{array}{c}\text { Date } \\
\text { (YYWK) }\end{array}$ & $\begin{array}{r}0-1 \\
\mathrm{~m} \mathrm{~s}^{-1}\end{array}$ & $\begin{array}{r}1-2 \\
\mathrm{~m} \mathrm{~s}^{-1}\end{array}$ & $\begin{array}{r}2-3 \\
\mathrm{~m} \mathrm{~s}^{-1}\end{array}$ & $\begin{array}{r}3-4 \\
\mathrm{~m} \mathrm{~s}^{-1}\end{array}$ & $\begin{array}{r}4-5 \\
\mathrm{~m} \mathrm{~s}^{-1}\end{array}$ & $\begin{array}{r}5-6 \\
\mathrm{~m} \mathrm{~s}^{-1}\end{array}$ & $\begin{array}{r}6-7 \\
\mathrm{~m} \mathrm{~s}^{-1}\end{array}$ & $\begin{array}{r}7-8 \\
\mathrm{~m} \mathrm{~s}^{-1}\end{array}$ & $\begin{array}{r}>8 \\
\mathrm{~m} \mathrm{~s}^{-1}\end{array}$ \\
\hline 8830 & -0.424 & -0.224 & -0.155 & -0.103 & -0.125 & -0.094 & -0.176 & -0.271 & -0.120 \\
8831 & -0.154 & -0.094 & 0.000 & -0.079 & -0.127 & -0.143 & -0.227 & -0.214 & -0.245 \\
8832 & -0.157 & -0.201 & -0.126 & -0.162 & -0.195 & -0.201 & -0.157 & -0.162 & -0.228 \\
8833 & -0.145 & -0.207 & -0.063 & 0.021 & -0.079 & -0.100 & -0.087 & -0.101 & -0.128 \\
8834 & -0.049 & -0.155 & 0.013 & -0.023 & -0.111 & -0.184 & -0.115 & -0.082 & -0.013 \\
8835 & -0.378 & -0.180 & -0.081 & -0.026 & -0.082 & -0.103 & -0.125 & -0.179 & -0.116 \\
8836 & -0.118 & -0.158 & -0.130 & -0.081 & -0.106 & -0.188 & -0.193 & -0.098 & -0.154 \\
8837 & -0.144 & -0.317 & -0.239 & -0.126 & -0.131 & -0.160 & -0.189 & -0.076 & -0.064 \\
8838 & -0.464 & -0.353 & -0.149 & -0.102 & -0.181 & -0.128 & -0.072 & -0.072 & -0.102 \\
8839 & 0.007 & -0.005 & -0.020 & 0.044 & -0.075 & -0.110 & -0.069 & -0.126 & -0.171 \\
8840 & -0.005 & -0.191 & -0.109 & -0.051 & -0.074 & -0.039 & -0.105 & -0.140 & -0.109 \\
8841 & - & -0.168 & -0.194 & -0.158 & -0.152 & -0.176 & -0.159 & -0.138 & -0.147 \\
8842 & -0.111 & -0.134 & -0.045 & 0.005 & 0.025 & -0.001 & -0.019 & -0.043 & -0.044 \\
8843 & - & - & -0.079 & -0.076 & -0.102 & -0.055 & -0.057 & -0.102 & -0.126 \\
& & & & & & & & & \\
Total & -0.166 & -0.184 & -0.076 & -0.052 & -0.106 & -0.126 & -0.133 & -0.143 & -0.144 \\
\hline
\end{tabular}


Table 22: Wind Speed Distribution

Daytime Data (Greater than $5 \mathrm{Wm}^{-2}$ )

\begin{tabular}{crrrrrrrrrr}
$\begin{array}{c}\text { Date } \\
(\mathrm{YYWK})\end{array}$ & $\begin{array}{r}0-1 \\
\mathrm{~m} \mathrm{~s}^{-1}\end{array}$ & $\begin{array}{r}1-2 \\
\mathrm{~m} \mathrm{~s}^{-1}\end{array}$ & $\begin{array}{r}2-3 \\
\mathrm{~m} \mathrm{~s}^{-1}\end{array}$ & $\begin{array}{r}3-4 \\
\mathrm{~m} \mathrm{~s}^{-1}\end{array}$ & $\begin{array}{r}4-5 \\
\mathrm{~m} \mathrm{~s}^{-1}\end{array}$ & $\begin{array}{r}5-6 \\
\mathrm{~m} \mathrm{~s}^{-1}\end{array}$ & $\begin{array}{r}6-7 \\
\mathrm{~m} \mathrm{~s}^{-1}\end{array}$ & $\begin{array}{r}7-8 \\
\mathrm{~m} \mathrm{~s}^{-1}\end{array}$ & $\begin{array}{r}>8 \\
\mathrm{~m} \mathrm{~s}^{-1}\end{array}$ & Total \\
\hline 8830 & 7 & 60 & 120 & 104 & 100 & 109 & 82 & 50 & 10 & 642 \\
8831 & 29 & 91 & 110 & 69 & 76 & 107 & 73 & 75 & 166 & 796 \\
8832 & 2 & 48 & 38 & 58 & 95 & 151 & 125 & 103 & 156 & 776 \\
8833 & 26 & 87 & 89 & 93 & 83 & 81 & 82 & 40 & 181 & 762 \\
8834 & 34 & 89 & 169 & 167 & 103 & 59 & 46 & 35 & 47 & 749 \\
8835 & 4 & 69 & 132 & 141 & 150 & 76 & 62 & 51 & 39 & 724 \\
8836 & 6 & 35 & 98 & 135 & 113 & 81 & 37 & 47 & 152 & 704 \\
8837 & 3 & 34 & 57 & 134 & 174 & 119 & 54 & 22 & 99 & 696 \\
8838 & 12 & 49 & 52 & 114 & 97 & 56 & 60 & 48 & 79 & 567 \\
8839 & 3 & 27 & 121 & 158 & 78 & 40 & 45 & 50 & 139 & 661 \\
8840 & 1 & 8 & 37 & 154 & 132 & 99 & 93 & 35 & 63 & 622 \\
8841 & 0 & 3 & 11 & 41 & 91 & 121 & 128 & 36 & 196 & 627 \\
8842 & 5 & 22 & 46 & 144 & 125 & 96 & 49 & 25 & 84 & 596 \\
8843 & 0 & 0 & 13 & 35 & 28 & 41 & 22 & 33 & 185 & 357 \\
& & & & & & & & & & \\
Total & 132 & 622 & 1093 & 1547 & 1445 & 1236 & 958 & 650 & 1596 & 9279 \\
\hline
\end{tabular}




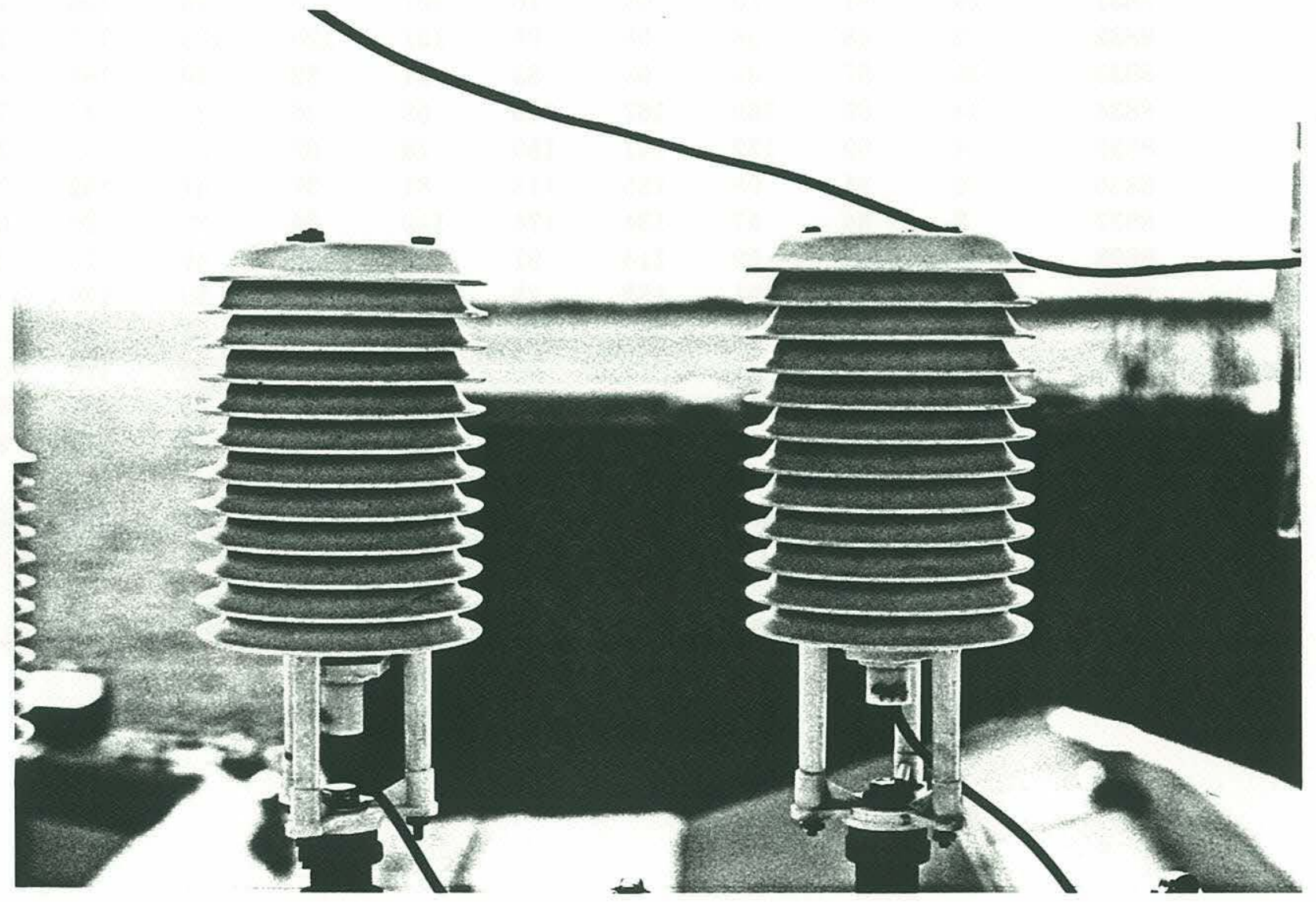

Figure 1: R. M. Young Gill Multi-Plate Radiation Shield (41002). 


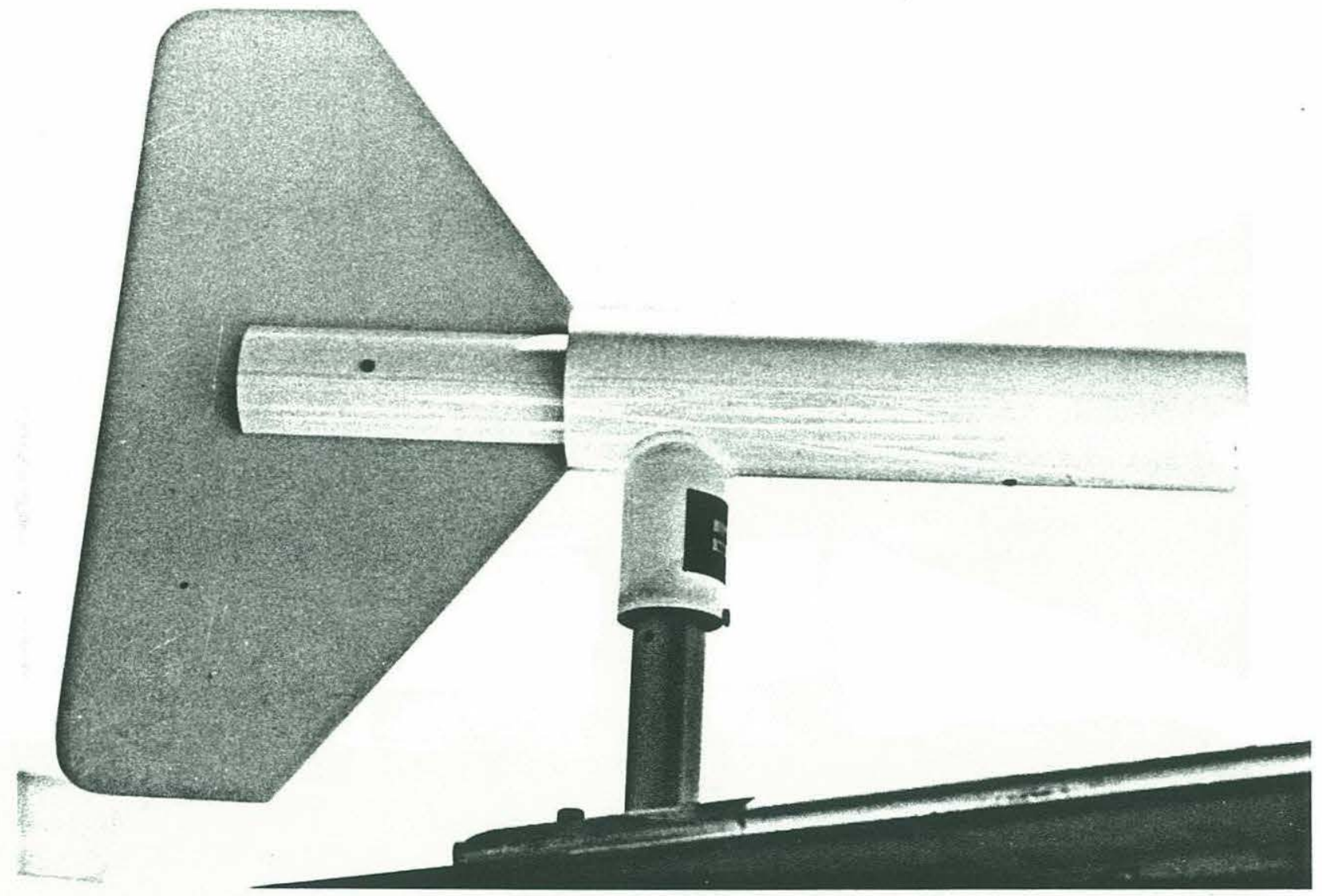

Figure 2: Met One Vane Aspirated Radiation Shield (071A). 


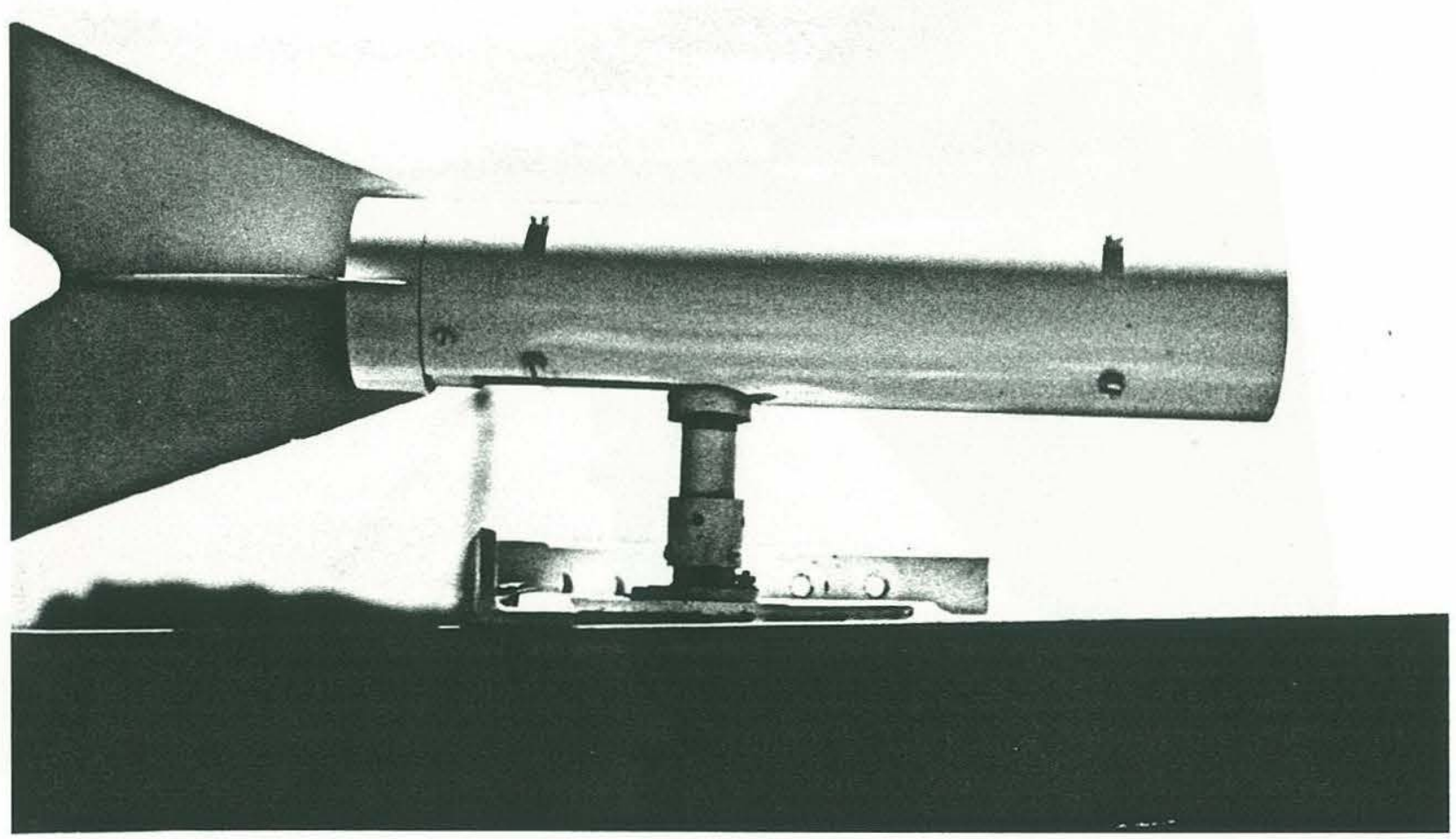

Figure 3: WHOI Vane Aspirated Radiation Shield (Developed by J. Dean). 


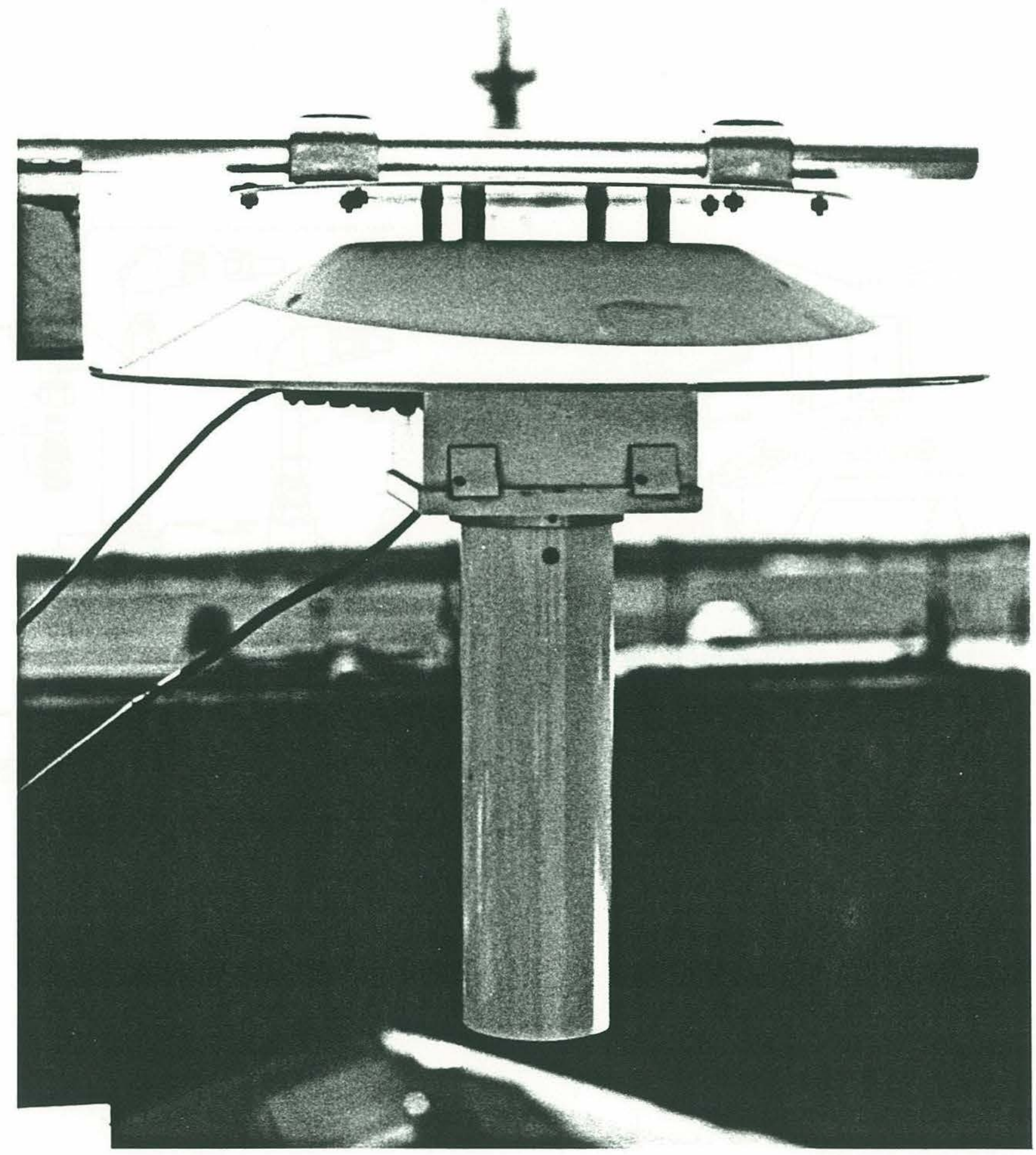

Figure 4: Met One Fan Aspirated Radiation Shield (076). 


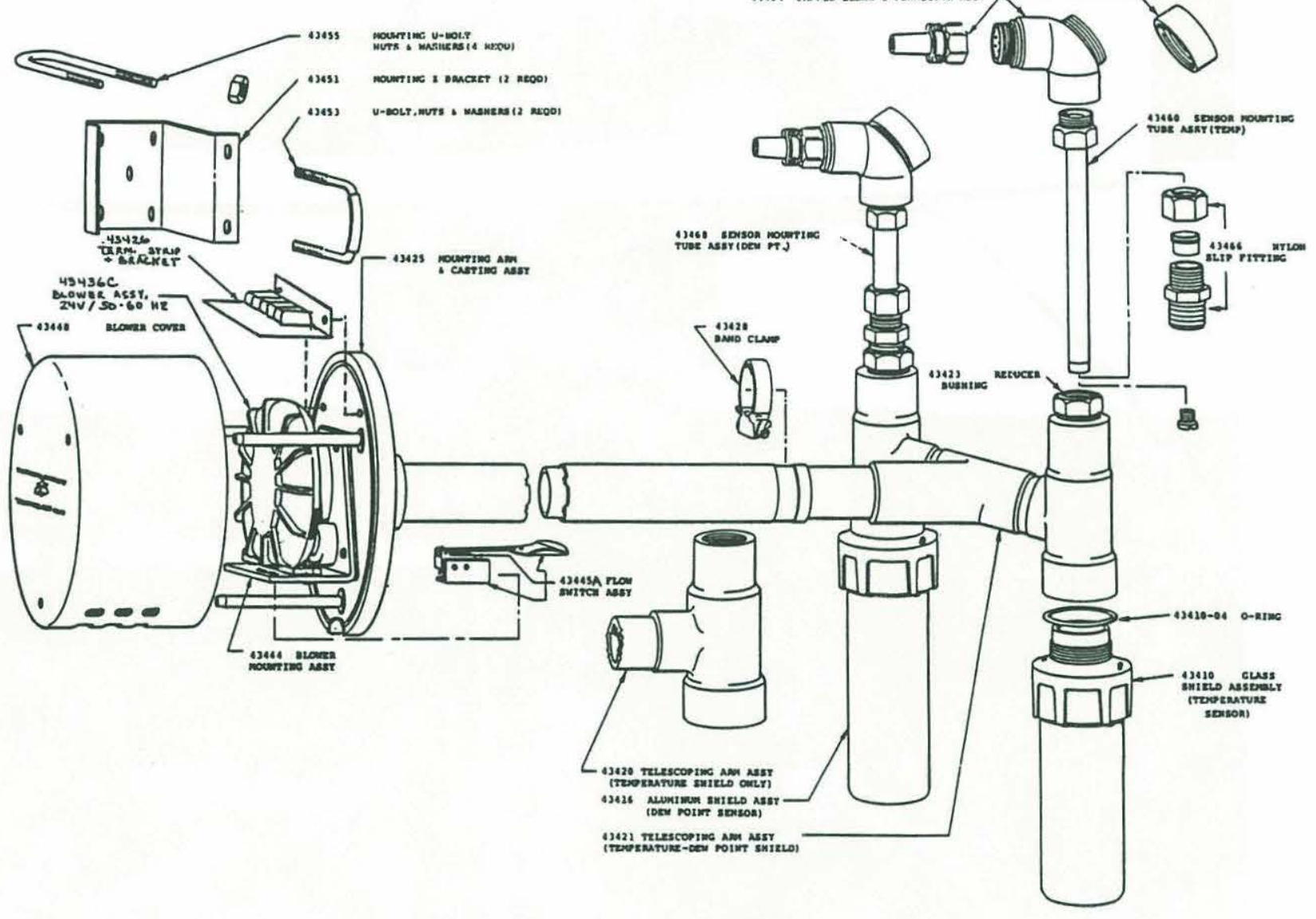

Figure 5: R. M. Young Gill Aspirated Radiation Shield (43404). 

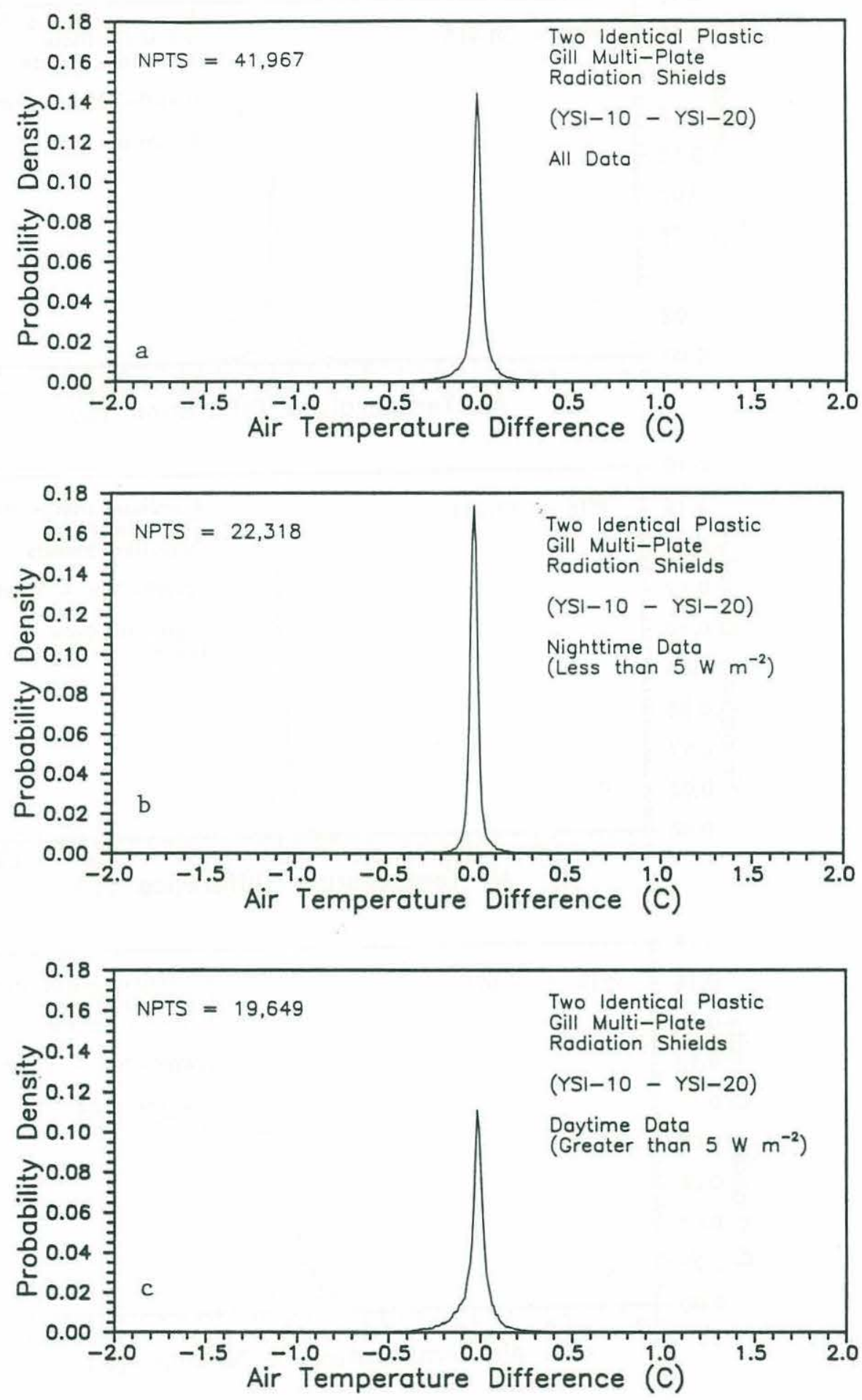

Figure 6: Probability density of air temperature difference between two identical plastic Gill multi-plate radiation shields for (a) all data, (b) nighttime data (insolation less than $5 \mathrm{~W} \mathrm{~m}^{-2}$ ), and (c) daytime data (insolation greater than $5 \mathrm{~W} \mathrm{~m}^{-2}$ ). 

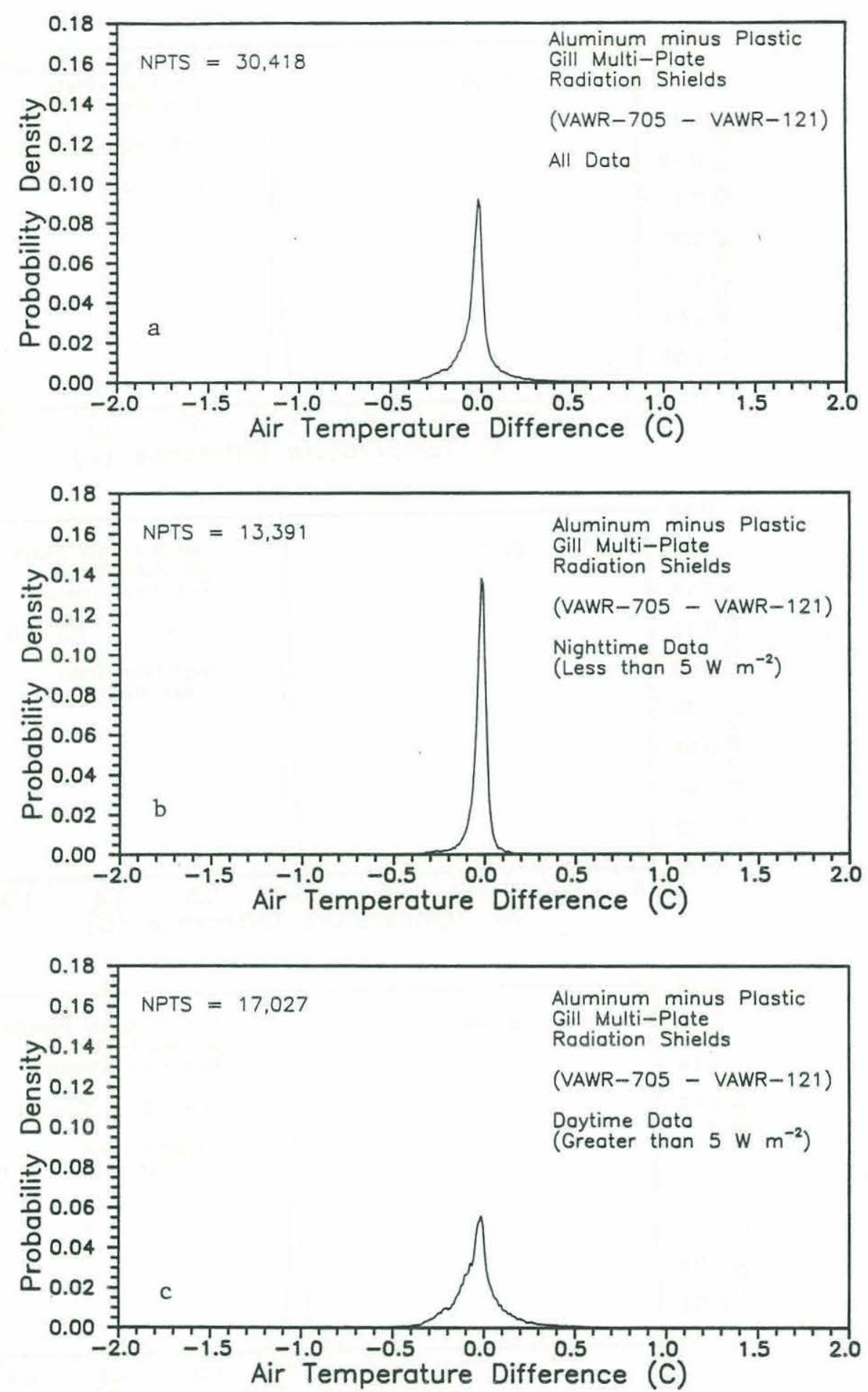

Figure 7: Probability density of air temperature difference between an aluminum and plastic multi-plate radiation shield for (a) all data, (b) nighttime data (insolation less than $5 \mathrm{~W} \mathrm{~m}^{-2}$ ), and (c) daytime data (insolation greater than $5 \mathrm{~W} \mathrm{~m}^{-2}$ ). 

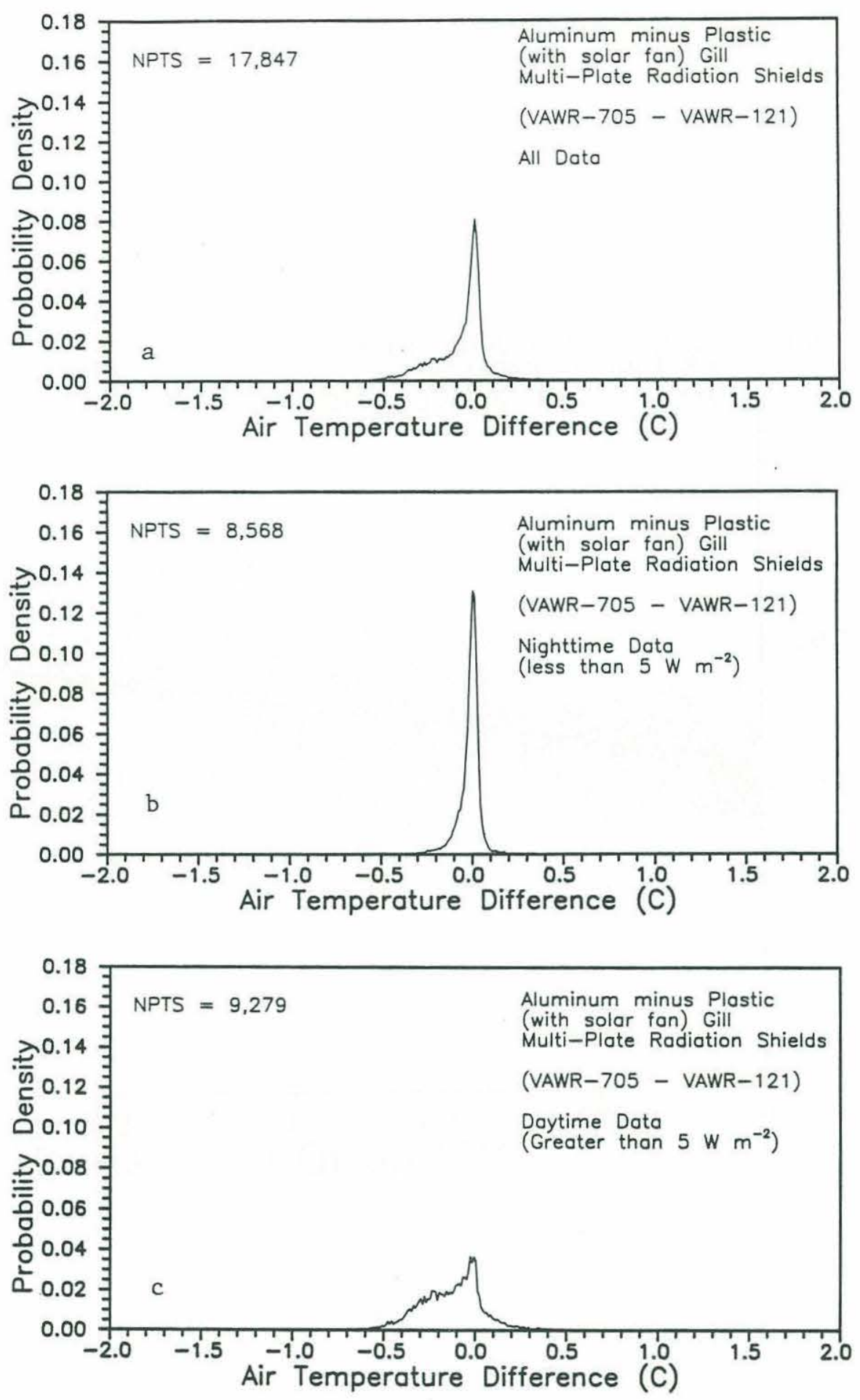

Figure 8: Probability density of air temperature difference between an aluminum and plastic (with solar powered fan) multi-plate radiation shield for (a) all data, (b) nighttime data (insolation less than $5 \mathrm{~W} \mathrm{~m}^{-2}$ ), and (c) daytime data (insolation greater than $\left.5 \mathrm{~W} \mathrm{~m}^{-2}\right)$. 


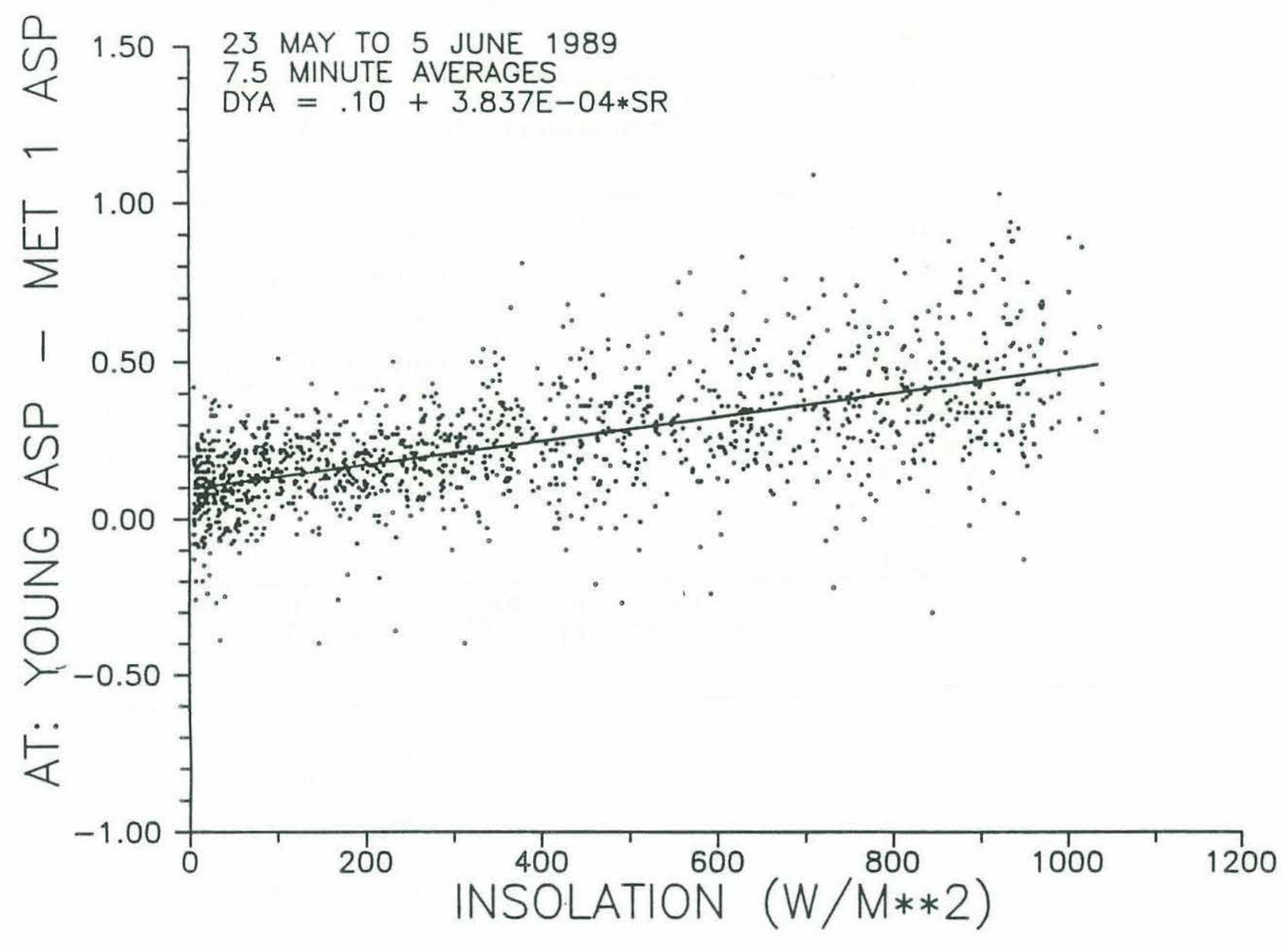

Figure 9: Scatter plot of R. M. Young Gill Aspirated minus Met One Aspirated air temperature as a function of insolation. 


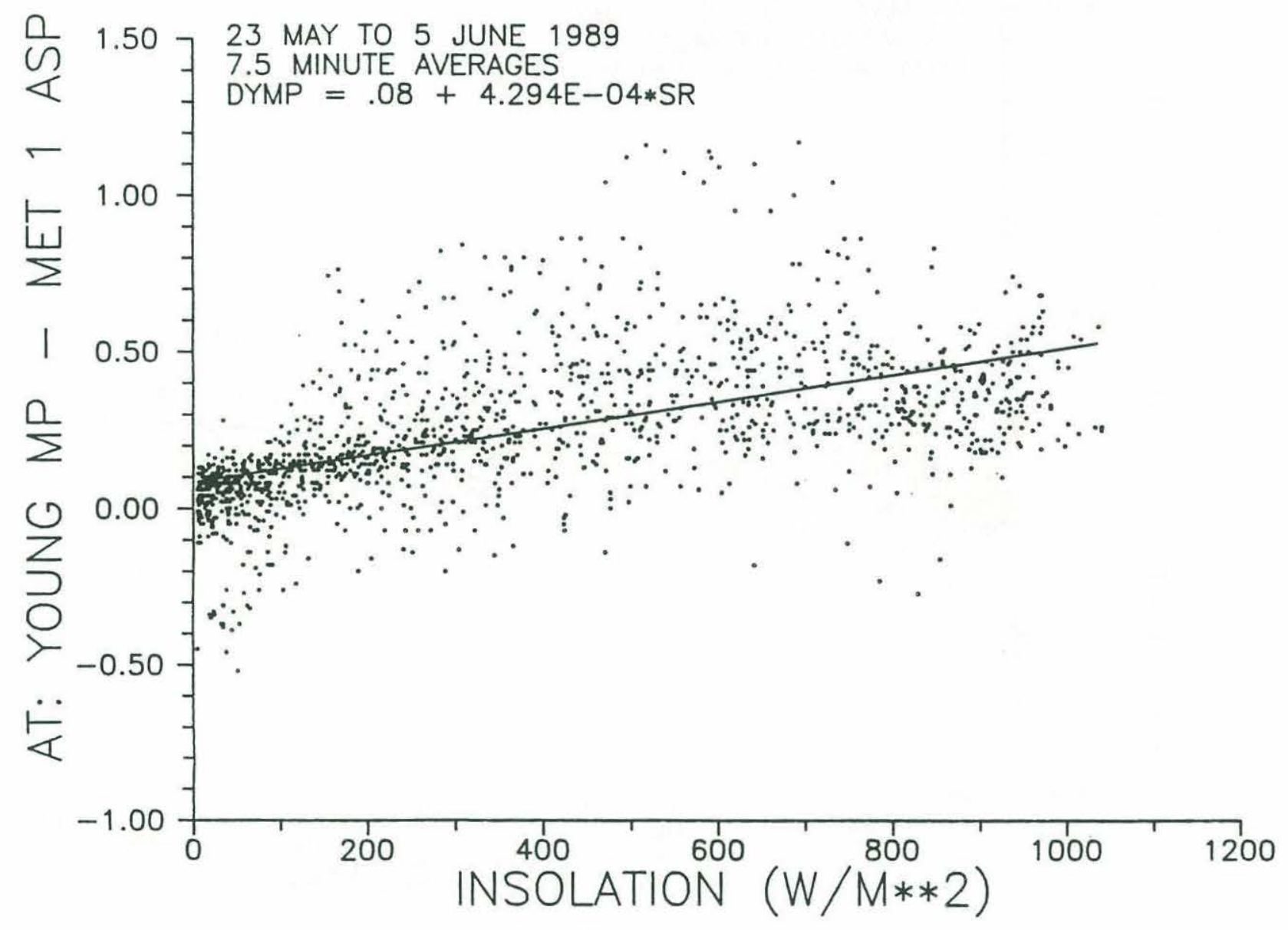

Figure 10: Scatter plot of R. M. Young Gill multi-plate minus Met One Aspirated air temperature as a function of insolation. 


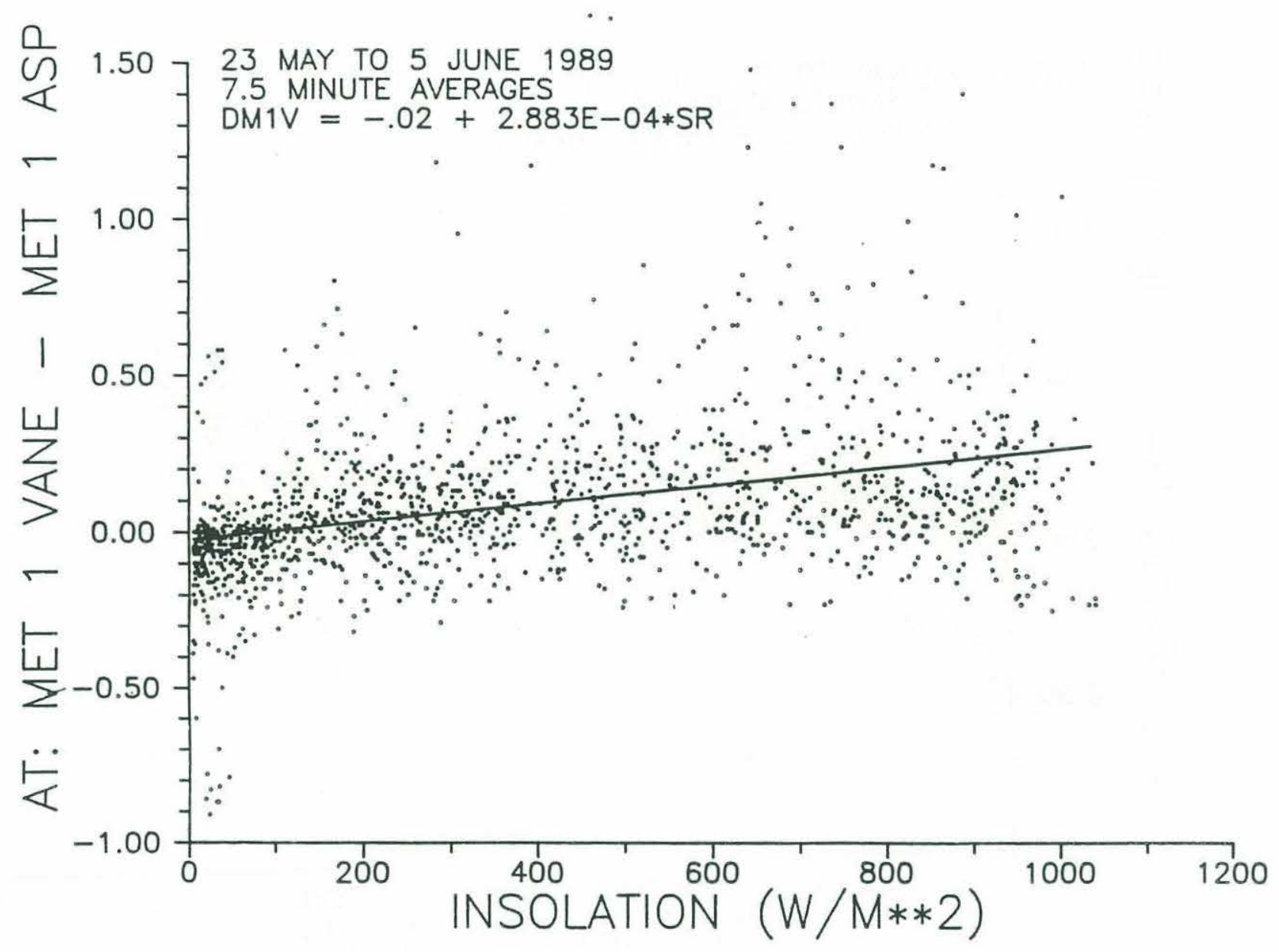

Figure 11: Scatter plot of Met One vane minus Met One Aspirated air temperature as a function of insolation. 


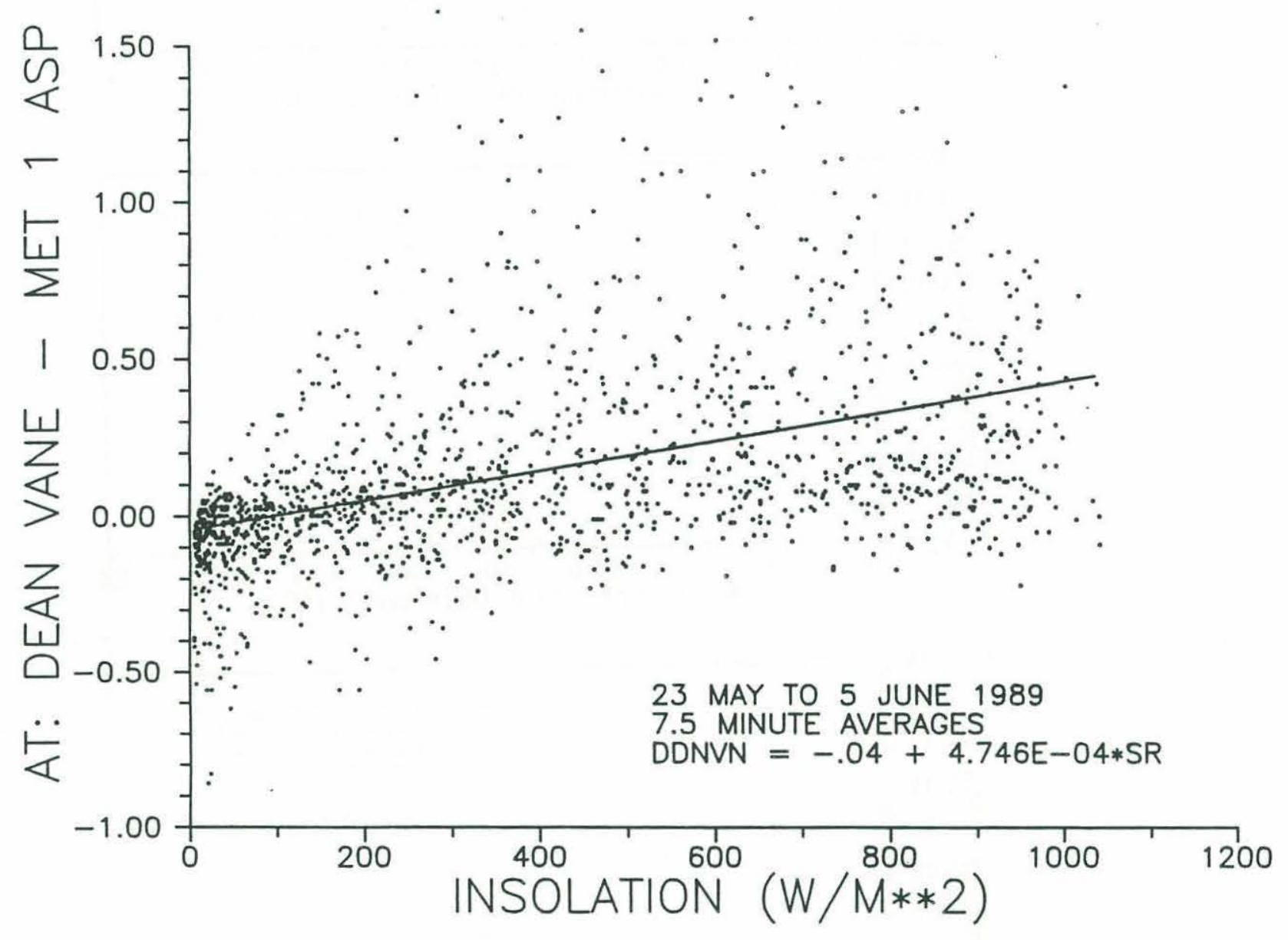

Figure 12: Scatter plot of WHOI vane minus Met One Aspirated air temperature as a function of insolation. 

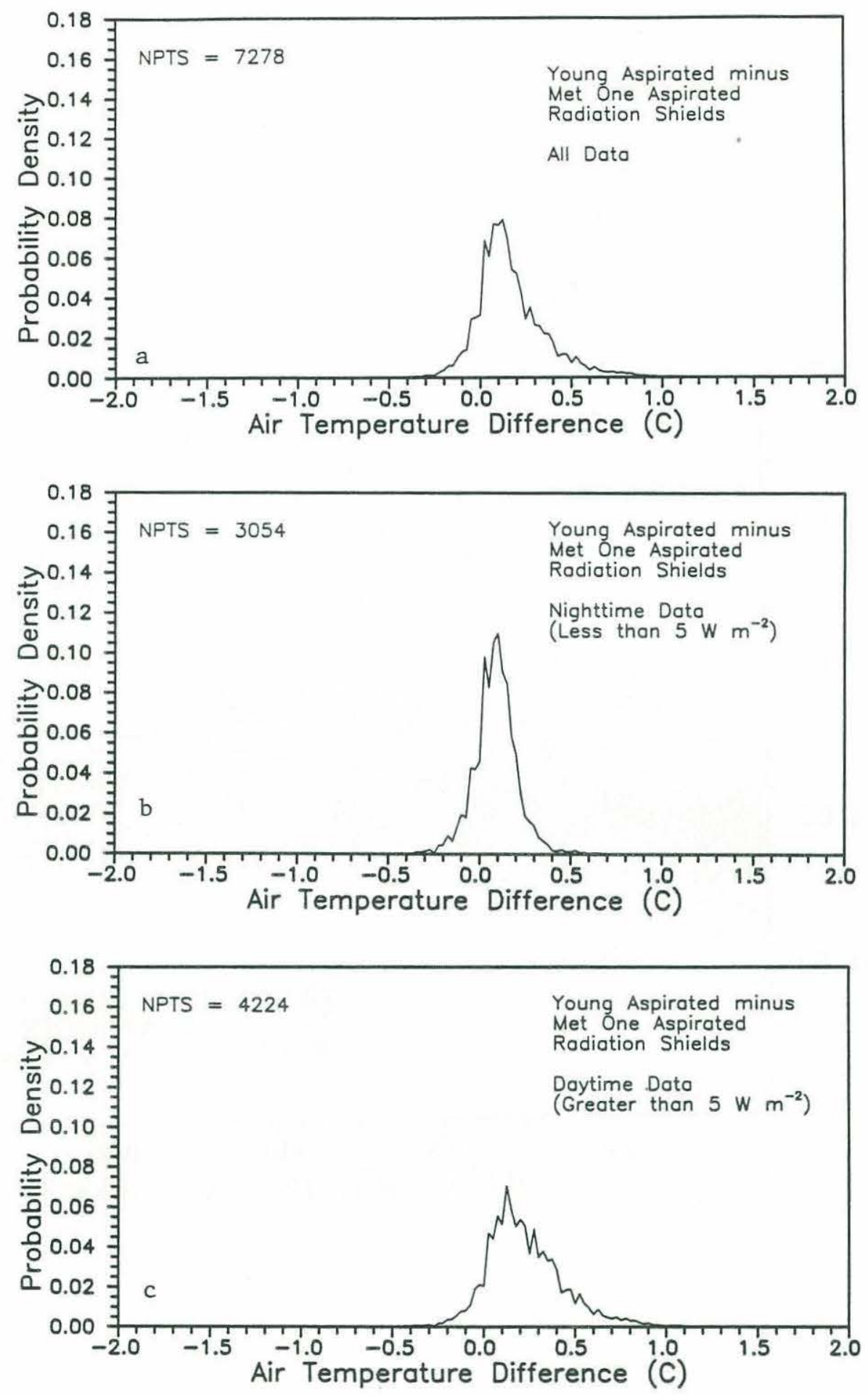

Figure 13: Probability density of R. M. Young Aspirated minus Met One Aspirated air temperature for (a) all data, (b) nighttime data, and (c) daytime data. 

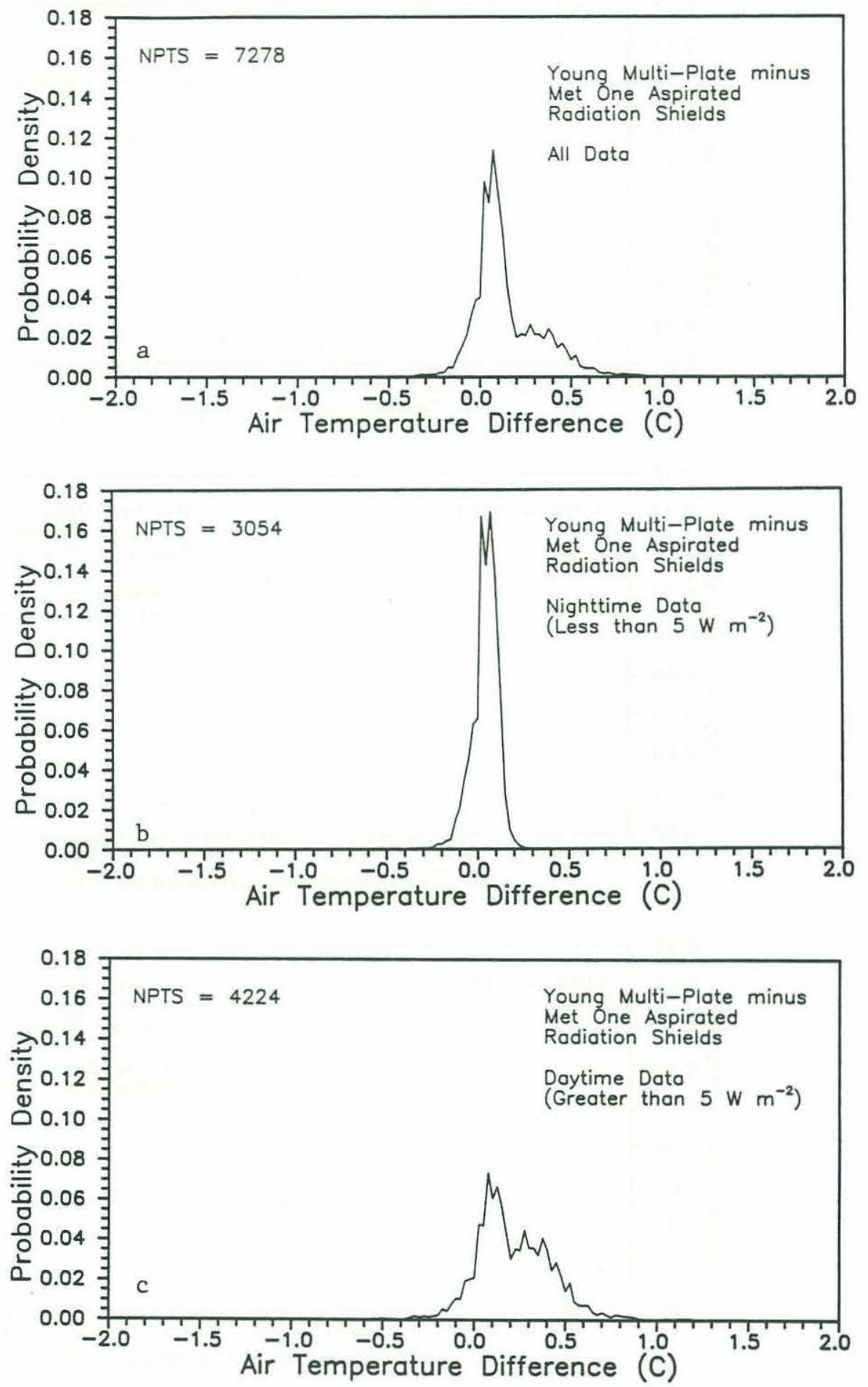

Figure 14: Probability density of R. M. Young multi-plate minus Met One Aspirated air temperature for (a) all data, (b) nighttime data, and (c) daytime data. 

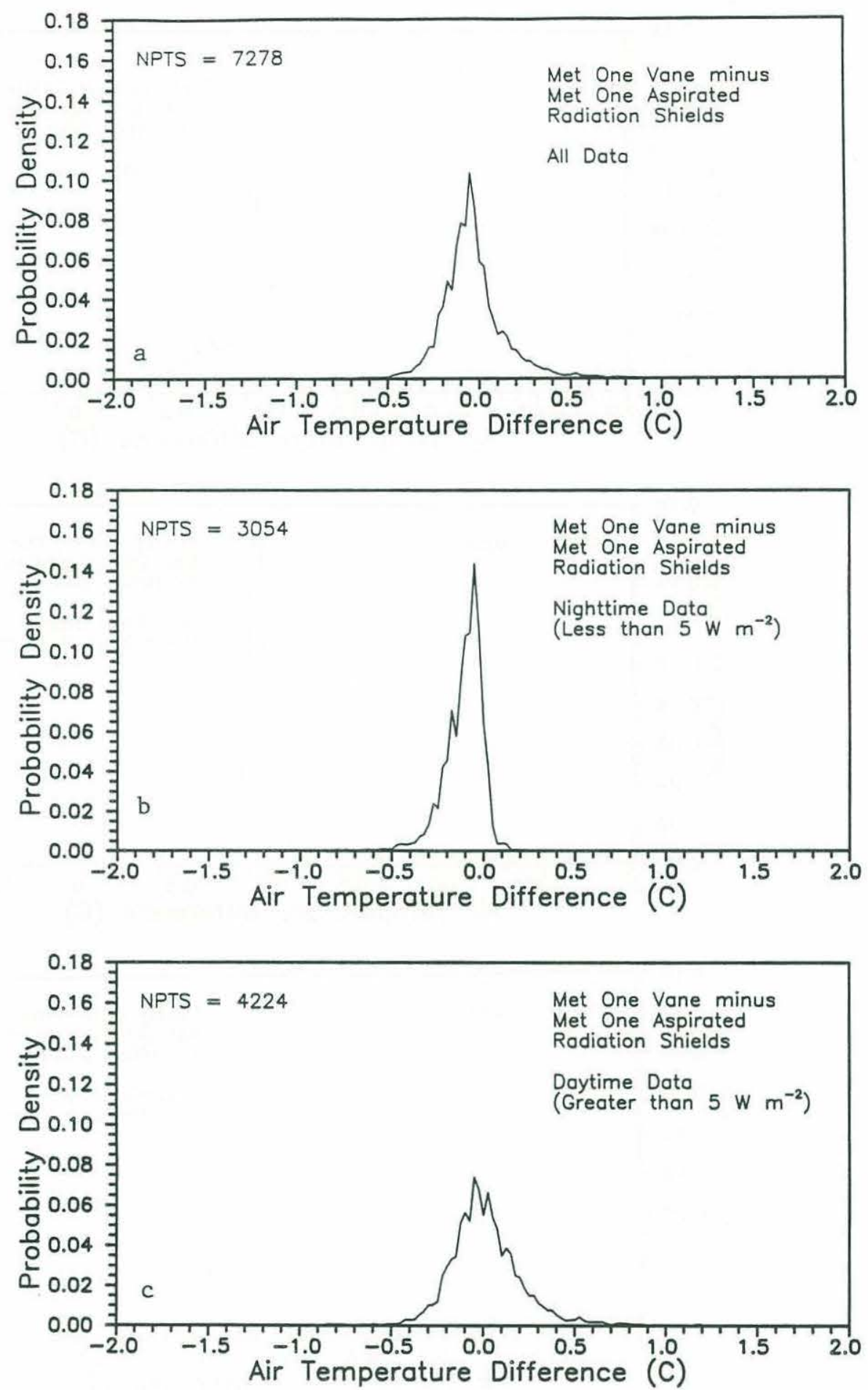

Figure 15: Probability density of Met One vane minus Met One Aspirated air temperature for (a) all data, (b) nighttime data, and (c) daytime data. 

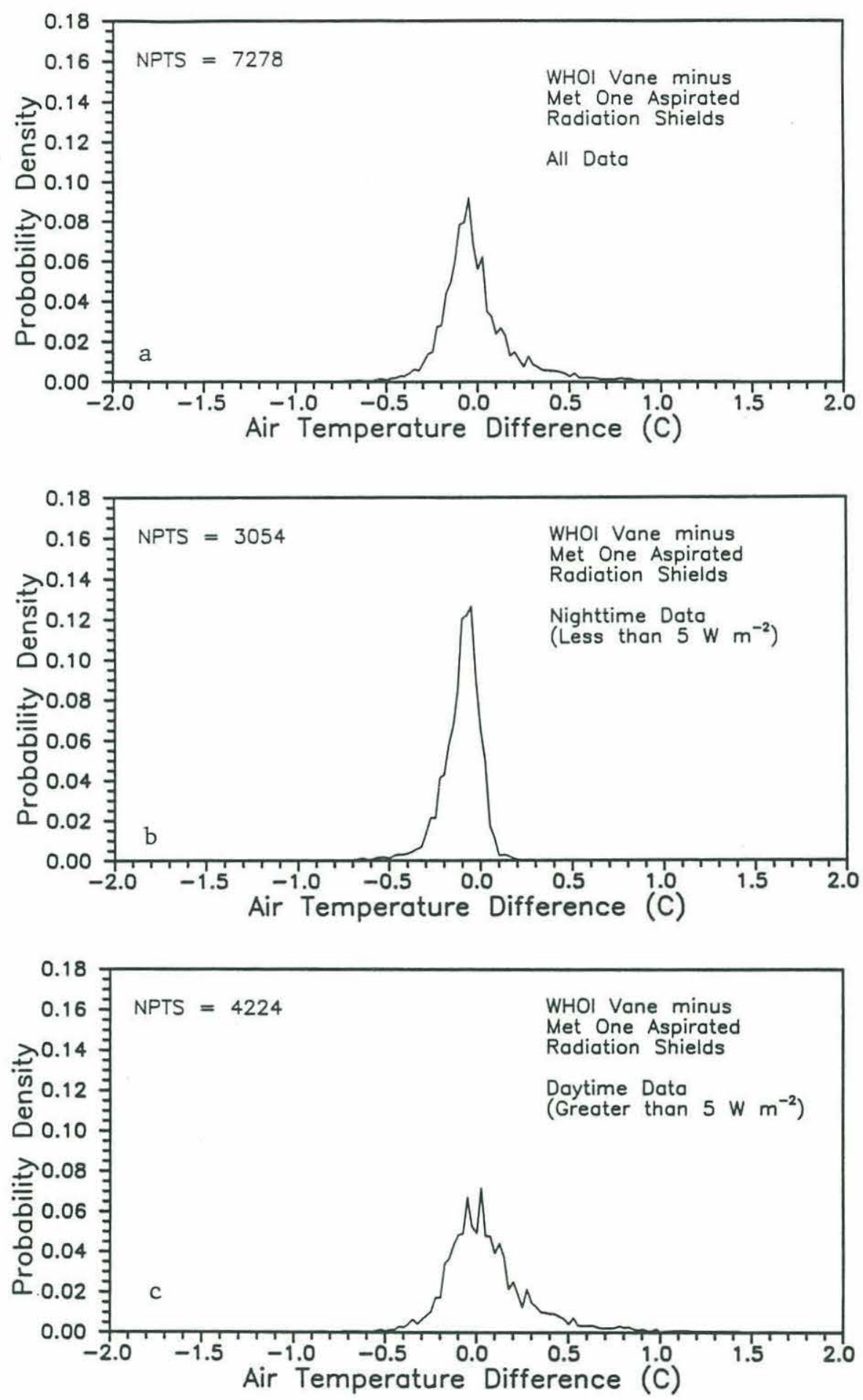

Figure 16: Probability density of WHOI Vane minus Met One Aspirated air temperature for (a) all data, (b) nighttime data, and (c) daytime data. 


\section{DOCUMENT LIBRARY}

July 5, 1989

Distribution List for Technical Report Exchange

Attn: Stella Sanchez-Wade

Documents Section

Scripps Institution of Oceanography

Library, Mail Code C-075C

La Jolla, CA 92093

Hancock Library of Biology \&

Oceanography

Alan Hancock Laboratory

University of Southern California

University Park

Los Angeles, CA 90089-0371

Gifts \& Exchanges

Library

Bedford Institute of Oceanography

P.O. Box 1006

Dartmouth, NS, B2Y 4A2, CANADA

Office of the International

Ice Patrol

c/o Coast Guard R \& D Center

Avery Point

Groton, CT 06340

Library

Physical Oceanographic Laboratory

Nova University

8000 N. Ocean Drive

Dania, FL 33304

NOAA/EDIS Miami Library Center 4301 Rickenbacker Causeway

Miami, FL 33149

Library

Skidaway Institute of Oceanography

P.O. Box 13687

Savannah, GA 31416

Institute of Geophysics

University of Hawaii

Library Room 252

2525 Correa Road

Honolulu, HI 96822

Library

Chesapeake Bay Institute

4800 Atwell Road

Shady Side, MD 20876

MIT Libraries

Serial Journal Room 14E-210

Cambridge, MA 02139
Director, Ralph M. Parsons Laboratory

Room 48-311

MIT

Cambridge, MA 02139

Marine Resources Information Center

Building E38-320

MIT

Cambridge, MA 02139

Library

Lamont-Doherty Geological Observatory

Colombia University

Palisades, NY 10964

Library

Serials Department

Oregon State University

Corvallis, OR 97331

Pell Marine Science Library

University of Rhode Island

Narragansett Bay Campus

Narragansett, RI 02882

Working Collection

Texas A\&M University

Dept. of Oceanography

College Station, TX 77843

Library

Virginia Institute of Marine Science

Gloucester Point, VA 23062

Fisheries-Oceanography Library

151 Oceanography Teaching Bldg.

University of Washington

Seattle, WA 98195

Library

R.S.M.A.S.

University of Miami

4600 Rickenbacker Causeway

Miami, FL 33149

Maury Oceanographic Library

Naval Oceanographic Office

Bay St. Louis

NSTL, MS 39522-5001

Marine Sciences Collection

Mayaguez Campus Library

University of Puerto Rico

Mayagues, Puerto Rico 00708 


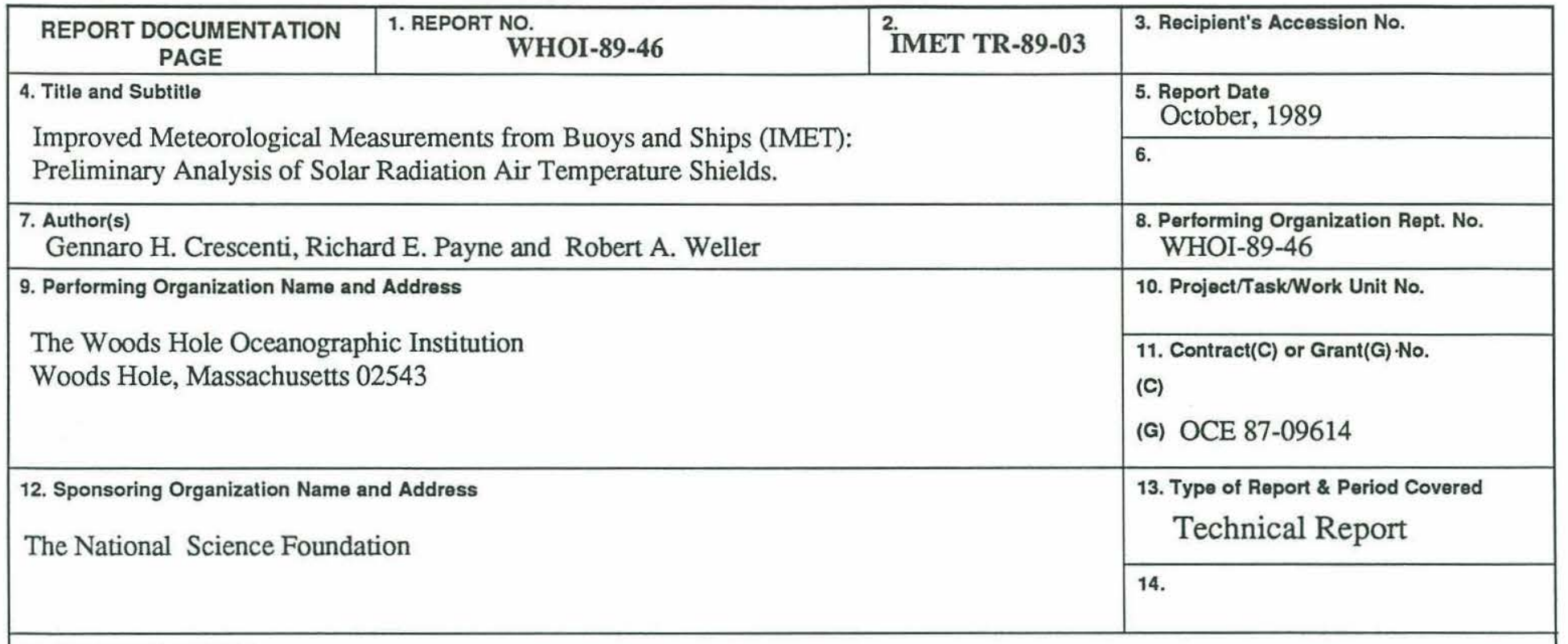

15. Supplementary Notes

This report should be cited as: Woods Hole Oceanog. Inst. Tech. Rept., WHOI-89-46, IMET TR-89-03

16. Abstract (Limit: 200 words)

Several different types of solar radiation air temperature shields are evaluated for use at sea on ships and buoys. They include three types of static or Thaller shields, two vane oriented shields, and two fan ventilated shields. A preliminary data analysis is presented and discussed.

17. Document Analysis a. Descriptors

1. radiation shields

2. marine meteorological measurements

3. ship and buoy instrumentation

b. Identifiers/Open-Ended Terms

c. COSATI Field/Group

18. Availability Statement

Approved for publication; distribution unlimited.

\begin{tabular}{|l|l|}
$\begin{array}{c}\text { 19. Security Class (This Report) } \\
\text { UNCLASSIFIED }\end{array}$ & $\begin{array}{c}\text { 21. No. of Pages } \\
53\end{array}$ \\
\hline 20. Security Class (This Page) & 22. Price \\
\hline
\end{tabular}

